\title{
Rigorous numerics for symmetric connecting orbits: even homoclinics of the Gray-Scott equation
}

\author{
Jan Bouwe van den Berg* \\ Jason D. Mireles-James ${ }^{\dagger}$ \\ Jean-Philippe Lessard ${ }^{\ddagger}$ \\ Konstantin Mischaikow ${ }^{\S}$
}

October 14, 2010

\begin{abstract}
In this paper we propose a rigorous numerical technique for the computation of symmetric connecting orbits for ordinary differential equations. The idea is to solve a projected boundary value problem (BVP) in a function space via a fixed point argument. The formulation of the projected BVP involves a high-order parameterization of the invariant manifolds at the steady states. Using this parameterization, one can obtain explicit exponential asymptotic bounds for the coefficients of the expansion of the manifolds. Combining these bounds with piecewise linear approximations, one can construct a contraction in a function space whose unique fixed point corresponds to the wanted connecting orbit. We have implemented the method to demonstrate its effectiveness, and we have used it to prove the existence of a family of even homoclinic orbits for the Gray-Scott system.
\end{abstract}

\section{Introduction}

Equilibria, periodic orbits, connecting orbits and more generally invariant manifolds are the fundamental components through which much of the structure of the dynamics of nonlinear differential equations is explained. Thus it is not surprising that there is a vast literature on numerical techniques for approximating

*VU University Amsterdam, Department of Mathematics, De Boelelaan 1081, 1081 HV Amsterdam, The Netherlands.

${ }^{\dagger}$ Rutgers University, Department of Mathematics, 110 Frelinghuysen Rd, Piscataway, NJ 08854, USA.

$\ddagger$ Rutgers University, Department of Mathematics,110 Frelinghuysen Rd, Piscataway, NJ 08854, USA and BCAM - Basque Center for Applied Mathematics, Bizkaia Technology Park, 48160 Derio, Bizkaia, Spain

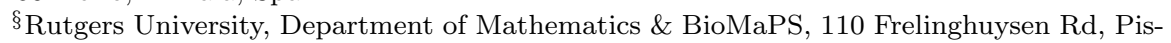
cataway, NJ 08854, USA. 
these objects. In particular, the last thirty years has witnessed a strong interest in developing computational methods for connecting orbits [5, 10, 12, 14, 15, 21]. As mentioned in [13], most algorithms for computing heteroclinic or homoclinic orbits reduce the question to solving a boundary value problem on a finite interval where the boundary conditions are given in terms of linear or higher order approximations of invariant manifolds near the steady states. We adopt the same philosophy in this paper. The novelty of our approach is that our computational techniques provide existence results and bounds on approximations that are mathematically rigorous. We hasten to add that a variety of authors have already developed methods that involve a combination of interval arithmetic with analytical and topological tools and provide proofs for the existence of homoclinic and heteroclinic solutions to differential equations [29, 24, 33, 6, 34]. However, the combination of techniques we propose appears to be unique, perhaps because our approach is being developed with additional goals in mind. We return to this point later.

For the sake of clarity in this paper we have chosen to restrict our attention to proving the existence of symmetric connecting orbits for systems of coupled second order equations

$$
\frac{d^{2} u}{d \tau^{2}}=\Psi(u)
$$

where $u \in \mathbb{R}^{n}$. Rescaling time by a factor $L>0$, leads to

$$
\frac{d^{2} u}{d t^{2}}=u^{\prime \prime}=L^{2} \Psi(u)
$$

with the boundary conditions

$$
\lim _{t \rightarrow \pm \infty} u(t)=u^{ \pm} \in \mathbb{R}^{n} .
$$

For simplicity we represent the symmetry condition by

$$
\mathcal{G}\left(u(0), u^{\prime}(0)\right)=0,
$$

where the case $\mathcal{G}\left(u(0), u^{\prime}(0)\right)=u^{\prime}(0)$ corresponds to looking for even homoclinic orbits and the case $\mathcal{G}\left(u(0), u^{\prime}(0)\right)=u(0)$ corresponds to looking for odd heteroclinic orbits when $\Psi(-u)=\Psi(u)$, but a mixture is also possible. The standard trick of defining $\tilde{u}=u^{\prime} \in \mathbb{R}^{n}$ reduces (2) to a $2 n$-dimensional first order system

$$
\left\{\begin{array}{l}
u^{\prime}=\tilde{u} \\
\tilde{u}^{\prime}=L^{2} \Psi(u) .
\end{array}\right.
$$

We assume that $\bar{y} \stackrel{\text { def }}{=}\left(u^{+}, 0\right) \in \mathbb{R}^{2 n}$ is a hyperbolic equilibrium for (4) with a stable manifold $W^{s}(\bar{y})$ of dimension $n$.

Observe that we have reduced the problem of looking for a symmetric connecting orbit to the afore mentioned boundary value problem:

$$
\left\{\begin{array}{l}
\frac{d^{2} u}{d t^{2}}=L^{2} \Psi(u(t)), \text { in }[0,1], \\
\mathcal{G}\left(u(0), u^{\prime}(0)\right)=0 \\
\left(u(1), u^{\prime}(1)\right) \in W^{s}(\bar{y}) .
\end{array}\right.
$$


There is a variety of ways in which one can obtain rigorous numerical approximations of the stable manifold, e.g. [36]. We make use of the parameterization method for invariant manifolds introduced in $[7,8,9]$. This method facilitates efficient, high order approximation of local stable and unstable manifolds associated with the hyperbolic directions of equilibria of vector fields. We present a concise, general introduction to the parameterization method that is meant to serve as a reference not only for the particular systems considered in this paper, but also for future applications. Theoretical aspects of the parameterization method are developed fully in $[7,8,9]$. However, an implementation of the numerical algorithms for stable/unstable manifolds of fixed points with dimension greater than one has appeared only in [27], and there only for fixed points of maps with complex conjugate stable/unstable eigenvalues. Thus, in Section 3 we develop all formulae, estimates, and arguments for finite but arbitrary dimensions.

In the context of the second order systems considered in this paper, the local parameterization of $W^{s}(\bar{y})$ is a function $P: \mathbb{R}^{n} \rightarrow \mathbb{R}^{2 n}$ of the form

$$
P(\theta)=\sum_{|\alpha| \geq 0} a_{\alpha} \theta^{\alpha}=\left(P^{(0)}(\theta), P^{(1)}(\theta)\right),
$$

with

$$
\begin{array}{rlrl}
a_{\alpha} & =\left(a_{\alpha}^{(0)}, a_{\alpha}^{(1)}\right) \in \mathbb{R}^{2 n}, \\
P^{(0)}(\theta) & =\sum_{|\alpha| \geq 0} a_{\alpha}^{(0)} \theta^{\alpha}, \quad & \left(a_{\alpha}^{(0)} \in \mathbb{R}^{n}\right), \\
P^{(1)}(\theta) & =\sum_{|\alpha| \geq 0} a_{\alpha}^{(1)} \theta^{\alpha}, & \left(a_{\alpha}^{(1)} \in \mathbb{R}^{n}\right),
\end{array}
$$

where we use the notation $\alpha=\left(\alpha_{1}, \ldots, \alpha_{n}\right) \in \mathbb{N}^{n},|\alpha|=\alpha_{1}+\ldots+\alpha_{n}, \theta=$ $\left(\theta_{1}, \ldots, \theta_{n}\right) \in \mathbb{R}^{n}$ and $\theta^{\alpha}=\theta_{1}^{\alpha_{1}} \cdot \ldots \cdot \theta_{n}^{\alpha_{n}}$.

Fixing $L>0$, problem (5) reduces to finding a solution $(\theta, u) \in \mathbb{R}^{n} \times$ $\left(C^{2}[0,1]\right)^{n}$ of the boundary value problem

$$
\left\{\begin{array}{l}
\frac{d^{2} u}{d t^{2}}=L^{2} \Psi(u), \text { in }[0,1], \\
\mathcal{G}\left(u(0), u^{\prime}(0)\right)=0, \\
u(1)=P^{(0)}(\theta), u^{\prime}(1)=P^{(1)}(\theta) .
\end{array}\right.
$$

As is made explicit in Section 2, problem (7) can be recast as a fixed point problem on $\mathbb{R}^{n} \times C[0,1]^{n}$. We adapt the concept of radii polynomials introduced in [11] to develop a computational technique that provides rigorous bounds and existence of the desired fixed point. The approximation scheme that underlies the estimates of [11] is based on spectral methods (Fourier series). For the boundary value problems that arise from the study of connecting orbits it appears that splines, which we use here, provide a more general and more straight 
forwardly applicable method. In this sense our efforts are strongly influenced by the results of $[28,35]$.

To demonstrate the effectiveness of our approach in Section 5 we apply our techniques to establish the existence of pulse solutions for the Gray-Scott system

$$
\left\{\begin{array}{l}
u_{1}^{\prime \prime}=u_{1} u_{2}^{2}-\lambda\left(1-u_{1}\right) \\
u_{2}^{\prime \prime}=\frac{1}{\gamma}\left(u_{2}-u_{1} u_{2}^{2}\right)
\end{array}\right.
$$

where $\lambda, \gamma$ are positive parameters. This equation models a continuously fed unstirred cubic autocatalytic reaction (see $[19,23]$ and references therein) and pulses (homoclinic orbits) represent stationary concentration patterns. By [23, Theorem A] for the set of parameter values

$$
\mathcal{C}_{0}=\{(\lambda, \gamma) \mid \lambda=1 / \gamma \text { and } 0<\gamma<2 / 9\},
$$

exact symmetric homoclinic solutions of $(8)$ for the steady-state $\left(u_{1}, u_{2}\right)=(1,0)$ are given by

$$
\bar{u}_{1}(x)=1-\frac{3 \gamma}{1+\zeta \cosh (x / \sqrt{\gamma})}, \quad \bar{u}_{2}(x)=\frac{3}{1+\zeta \cosh (x / \sqrt{\gamma})},
$$

where $\zeta=\left(1-\frac{9 \gamma}{2}\right)^{1 / 2}$. These homoclinics are stable under perturbations in the parameter values in the following sense. For a fixed $\bar{\gamma} \in\left(0, \frac{2}{9}\right)$ and for a fixed $\varepsilon_{0}>0$, define the line piece

$$
\mathcal{C}_{\varepsilon_{0}}(\bar{\gamma}) \stackrel{\text { def }}{=}\left\{(\lambda(\varepsilon), \gamma(\varepsilon))=\left(\frac{1+\varepsilon}{\bar{\gamma}}, \bar{\gamma}\right):|\varepsilon| \leq \varepsilon_{0}\right\} .
$$

For any given $(1 / \bar{\gamma}, \bar{\gamma}) \in \mathcal{C}_{0}$, it is known that there is an $\varepsilon_{0}=\varepsilon_{0}(\bar{\gamma})>0$ such that there exists a continuous branch of even homoclinic orbits $\left(u_{1}(\varepsilon), u_{2}(\varepsilon)\right)$ of (8) with $(\lambda(\varepsilon), \gamma(\varepsilon)) \in \mathcal{C}_{\varepsilon_{0}}(\bar{\gamma})$ such that $(\lambda(\varepsilon), \gamma(\epsilon))$ converges to $(1 / \bar{\gamma}, \bar{\gamma})$ and $\left(u_{1}(\varepsilon), u_{2}(\varepsilon)\right)$ converges to $\left(\bar{u}_{1}, \bar{u}_{2}\right)$ as $\varepsilon$ tends to 0 (see [23, Theorem C]).

Our approach is to fix $\bar{\gamma}=.15$ and to explore homoclinic solutions further away from the curve $\mathcal{C}_{0}$ of explicit solutions. Note that in the context of looking for even homoclinics of the Gray-Scott equation (8), the boundary value problem (7) becomes

$$
\left\{\begin{array}{l}
\frac{d^{2} u}{d t^{2}}=L^{2}\left(\begin{array}{c}
u_{1} u_{2}^{2}-\lambda\left(1-u_{1}\right) \\
\frac{1}{\gamma}\left(u_{2}-u_{1} u_{2}^{2}\right)
\end{array}\right), \text { in }[0,1], \\
u^{\prime}(0)=0, u(1)=P^{(0)}(\theta), u^{\prime}(1)=P^{(1)}(\theta),
\end{array}\right.
$$

with $u=\left(u_{1}, u_{2}\right)$. Here is an application of our novel rigorous computational method:

Theorem 1.1. Fix $\bar{\gamma}=0.15$, and define

$$
\sigma_{k} \stackrel{\text { def }}{=}\left\{\begin{array}{cl}
\frac{3 k}{200}, & k=-8, \ldots,-1 ; \\
0, & k=0 \\
\frac{3 k}{100}, & k=1, \ldots, 22
\end{array}\right.
$$


and

$$
\Sigma_{\bar{\gamma}} \stackrel{\text { def }}{=}\left\{\left(\frac{1+\sigma_{k}}{\bar{\gamma}}, \bar{\gamma}\right): k=-8, \ldots, 22\right\} .
$$

Let $L=1.2$ for $k>0$ and $L=1.7$ for $k<0$. Then, for every $(\lambda, \gamma) \in \Sigma_{\bar{\gamma}}$ there exists a small set $B=B_{k} \subset \mathbb{R}^{2} \times(C[0,1])^{2}$ containing a unique solution $\left(\tilde{\theta}, \tilde{u}_{1}, \tilde{u}_{2}\right)$ of problem $(10)$.

Corollary 1.2. For the parameter values $(\lambda, \gamma) \in \Sigma_{0.15}$ there exists a symmetric homoclinic orbits of $(8)$ to the steady state $\left(u_{1}, u_{2}\right)=(1,0)$.

The proof of Theorem 1.1 is found in Section 5. A geometric representation of the solutions obtained in Theorem 1.1 can be seen in Figure 1(a) and their corresponding parameters are depicted in Figure 1(b). Note that the construction of each set $B$ in Theorem 1.1 is done with the use of the radii polynomials presented in Section 2 and the analysis at the boundary values is carried out using the theory of the parametrization method introduced in Sections 3 and 4.

Before turning to the details of our approach, we discuss several of the longer term goals that lead to our particular choice of methods. The first goal is directly related to the opening comments of this paper. In the context of differential equations the simplest and best understood means of generating chaotic dynamics is through solutions that are homoclinic to periodic orbits. Our results on the Gray-Scott equation demonstrate the practicality of using these techniques to find orbits homoclinic to equilibria. There are two fundamental requirements to obtain results such as these for periodic orbits. First, one must be able to rigorously and accurately identify periodic orbits. The method of radii polynomials has proven effective for this task in a variety of settings [26, 2]. Second, one must be able to parameterize stable and unstable manifolds for the periodic orbits. Parameterization of stable/unstable manifolds associated with periodic orbits of vector fields is discussed from a theoretic prespective in [9], and implemented numerically in [20] for the phase resetting curves in biological systems. We are not aware of any applications of these methods to rigorous computer assisted proofs. The ideas developed in the present work should be extendable to the case of periodic asymptotic behaviour.

The second goal is clearly demonstrated by contrasting our computational results on pulse solutions to Gray-Scott with the perturbative results of [23]. In this paper we prove the existence of a finite number of homoclinic orbits at points on the line $\gamma=0.15$ in parameter space, as opposed to a continuous branch of solutions. More generally, most physical models have free parameters and it is of interest to be able to determined the dynamics over large sets of parameter values. Thus, the ability to efficiently and rigorously compute branches of solutions is highly desirable. There are theoretical results that have been implemented that justify why the method of radii polynomials provides an efficient means for computing branches of solutions [3]. The best way to extend these ideas to the setting of connecting orbits, in particular algorithms for obtaining parameterized families of stable and unstable manifolds, remains an open problem, but theoretical work [8] suggests that there are no fundamental mathematical obstacles. 


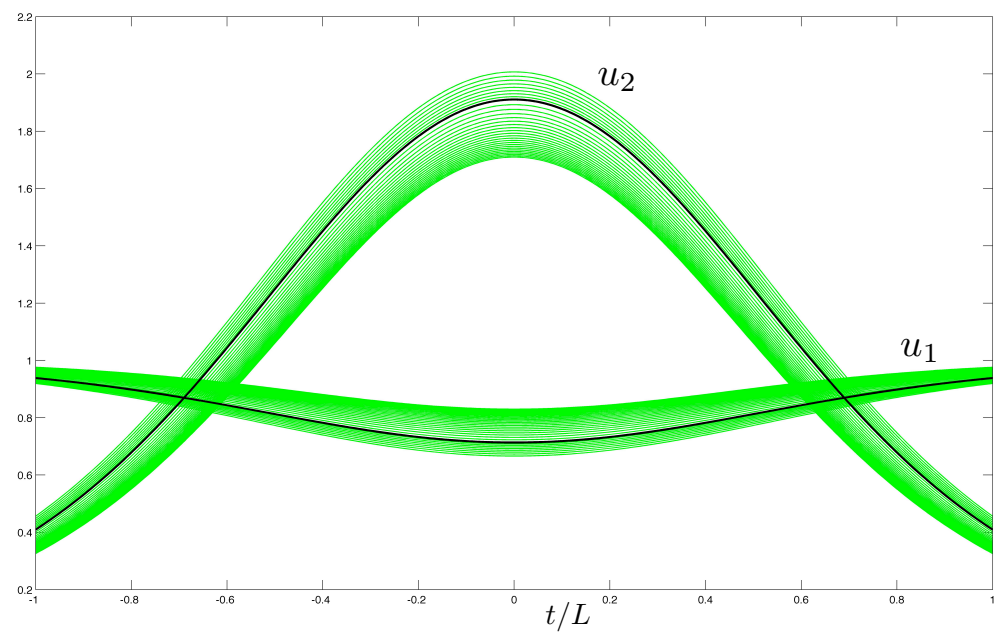

(a) Solution profiles. The explicit symmetric homoclinic orbit (9) from [23] for $\bar{\gamma}=0.15$ (in black) and the thirty rigorously computed homoclinic orbits of Theorem 1.1 (in green). Each solution couple $(u, v)$ has been extended by symmetry and is the center of a small set $B$ containing a genuine solution of problem (10).

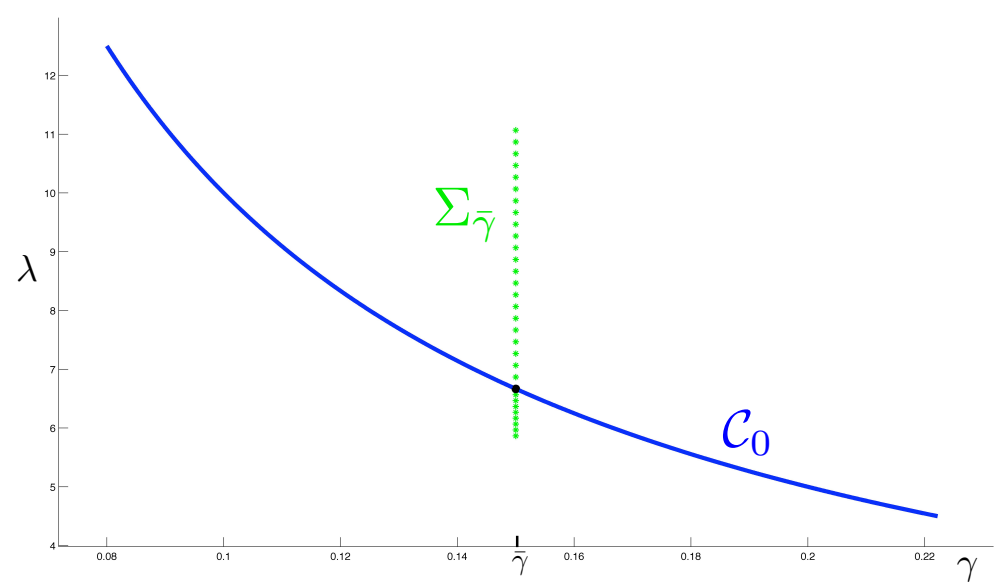

(b) Parameter space. The symmetric homoclinic orbit (9) from [23] for $\bar{\gamma}=0.15$ (in black) and the associated parameters of the thirty rigorously computed homoclinic orbits of Theorem 1.1 (in green).

Figure 1: Geometric representation of the solutions generated by Theorem 1.1. 
The final goal that we mention here involves rigorous computations for partial and functional differential equations. In this case the phase space for the dynamics is infinite dimensional. Again, the method of radii polynomials provides an effective technique for finding fixed points and periodic orbits in this setting $[26,16]$ even in the context of higher dimensional domains $[17,18]$. The challenging problem is to adapt the parameterization method to this setting in such a way that one can obtain rigorous computational results. It should be noted that the original work is developed under the assumption that the phase space is a Banach space [7]. However, it is assumed that the underlying dynamics is a flow, thus it is not directly applicable to parabolic equations. On the other hand, as is indicated by [7, Theorem 2.1] the theory is applicable to finite dimensional invariant submanifolds of the stable and unstable manifolds which is essential for a rigorous computational approach.

\section{Rigorous numerics for symmetric connecting orbits: formulation, fixed point and radii poly- nomials}

As is indicated in the Introduction we prove the existence of the connecting orbit by solving a fixed point problem. The first step in deriving the fixed point problem is presented in Section 2.1, where we recast the boundary value problem (7) as an equivalent problem of the form $F(\theta, u)=0$ on the space $X=\mathbb{R}^{n} \times C[0,1]^{n}$. The next step is to compute an approximate solution $\left(\hat{\theta}, \hat{u}_{h}\right)$ of $F=0$ using a finite dimensional reduction, see Section 2.2. One can then define a Newton-like operator $T$ around the approximate solution whose fixed points correspond to solutions of $F=0$. The proof of existence of the desired fixed point of $T$ is achieved using the concept of radii polynomials which are introduced in Section 2.3. Finally, we provide in Section 2.4 the explicit construction of the radii polynomials.

\subsection{Set up of the problem $F(\theta, u)=0$}

The goal of this section is to transform the boundary value problem (7) into one of the form $F(\theta, u)=0$ in the Banach space $\mathbb{R}^{n} \times C[0,1]^{n}$. We assume here that the boundary condition at $t=0$ is $\mathcal{G}\left(u(0), u^{\prime}(0)\right)=u^{\prime}(0)=0$, meaning that we are looking for even homoclinic orbits. The case $\mathcal{G}\left(u(0), u^{\prime}(0)\right)=u(0)=0$ (odd heteroclinic orbits) can be treated similarly (see Remark 2.2).

Integrating $\frac{d^{2} u}{d t^{2}}=L^{2} \Psi(u)$ component-wise between 0 and $t$, and using $u^{\prime}(0)=0$, results in

$$
u^{\prime}(t)=L^{2} \int_{0}^{t} \Psi(u(s)) d s .
$$

Setting $t=1$ and using the boundary conditions $u^{\prime}(1)=P^{(1)}(\theta)$ at the stable 
manifold, one obtains the $n$ conditions

$$
P^{(1)}(\theta)-L^{2} \int_{0}^{1} \Psi(u(s)) d s=0 \in \mathbb{R}^{n} .
$$

Now, integrating (11) between $t$ and 1 and using the boundary conditions $u(1)=$ $P^{(0)}(\theta)$, one finds

$$
\begin{aligned}
u(t) & =f(\theta, u)(t) \\
& \stackrel{\text { def }}{=} P^{(0)}(\theta)+(t-1) L^{2} \int_{0}^{t} \Psi(u(s)) d s+L^{2} \int_{t}^{1}(s-1) \Psi(u(s)) d s,
\end{aligned}
$$

where $f=\left(f_{1}, \ldots, f_{n}\right)$. Finally, appending (12) to equation (13), one defines $F: \mathbb{R}^{n} \times C[0,1]^{n} \rightarrow \mathbb{R}^{n} \times C[0,1]^{n}$ by

$$
F(\theta, u)=\left(\begin{array}{c}
P^{(1)}(\theta)-L^{2} \int_{0}^{1} \Psi(u(s)) d s \\
f(\theta, u)-u
\end{array}\right) .
$$

Remark 2.1. The solutions of $F(\theta, u)=0 \in \mathbb{R}^{n} \times C[0,1]^{n}$ given by (14) correspond to solutions of the boundary value problem $(7)$ with $\mathcal{G}\left(u(0), u^{\prime}(0)\right)=$ $u^{\prime}(0)$.

Remark 2.2. The case $\mathcal{G}\left(u(0), u^{\prime}(0)\right)=u(0)=0$ leads to the function

$$
\widetilde{F}(\theta, u)=\left(\begin{array}{c}
P^{(0)}(\theta)-u(1) \\
\tilde{f}(\theta, u)-u
\end{array}\right)
$$

where

$$
\tilde{f}(\theta, u)(t)=P^{(1)}(\theta) t-L^{2} \int_{0}^{t} s \Psi(u(s)) d s-L^{2} t \int_{t}^{1} \Psi(u(s)) d s .
$$

The solutions of $\widetilde{F}(\theta, u)=0 \in \mathbb{R}^{n} \times C[0,1]^{n}$ given by (15) correspond to solutions of the boundary value problem $(7)$ with $\mathcal{G}\left(u(0), u^{\prime}(0)\right)=u(0)$.

\subsection{Finite dimensional reduction}

Since $\mathbb{R}^{n} \times C[0,1]^{n}$ is an infinite dimensional space we cannot directly compute on (14), and thus we turn to the problem of deriving an appropriate finite dimensional approximation. We begin by discretizing the interval $[0,1]$ using the mesh

$$
\Delta_{h}=\left\{0=t_{0}<t_{1}<t_{2}<\cdots<t_{m}=1\right\},
$$

with the subscript denoting the finite mesh size. Using the above mesh, consider the subset $S_{h} \subset C[0,1]$ of piecewise linear functions (linear splines) defined on $\Delta_{h}$. We will identify $S_{h}$ and $\mathbb{R}^{m+1}$ whenever convenient. We define the projection

$$
\begin{aligned}
\Pi_{h}: C[0,1] & \rightarrow S_{h} \\
v & \mapsto \Pi_{h} v \stackrel{\text { def }}{=}\left(v_{0}, v_{1}, \ldots, v_{m}\right)
\end{aligned}
$$


where $v_{j}=v\left(t_{j}\right)$, for $j=0, \ldots, m$. For vector-valued functions $u$ we will use the short hand notation

$$
u_{h} \stackrel{\text { def }}{=}\left(\Pi_{h}\right)^{n} u \stackrel{\text { def }}{=}\left(\Pi_{h} u_{1}, \ldots, \Pi_{h} u_{n}\right) \in S_{h}^{n} .
$$

Note that using the projection one obtains the direct sum decomposition

$$
C[0,1]=\Pi_{h} C[0,1] \oplus\left(I-\Pi_{h}\right) C[0,1],
$$

where $I$ denotes the identity.

As is described in the Introduction the boundary conditions are given in terms of a local parameterization $P$ of the stable manifold that is expressed in terms of infinite series expansions (6). For the purpose of computations we can only work with a finite number of terms. Hence we choose a parameterization order $N \in \mathbb{N}$ and define

$$
P_{N}(\theta)=\left(P_{N}^{(0)}(\theta), P_{N}^{(1)}(\theta)\right) \stackrel{\text { def }}{=} \sum_{0 \leq|\alpha| \leq N} a_{\alpha} \theta^{\alpha}=\left(\sum_{0 \leq|\alpha| \leq N} a_{\alpha}^{(0)} \theta^{\alpha}, \sum_{0 \leq|\alpha| \leq N} a_{\alpha}^{(1)} \theta^{\alpha}\right) .
$$

Definition 2.3. The finite dimensional projection $F^{(m, N)}: \mathbb{R}^{n} \times\left(S_{h}\right)^{n} \rightarrow$ $\mathbb{R}^{n} \times\left(S_{h}\right)^{n}$ of $(14)$ is given by

$$
F^{(m, N)}\left(\theta, u_{h}\right)=\left(\begin{array}{c}
P_{N}^{(1)}(\theta)-L^{2} \int_{0}^{1} \Psi\left(u_{h}(s)\right) d s \\
f^{(m, N)}\left(\theta, u_{h}\right)-u_{h}
\end{array}\right),
$$

where each $f_{i}^{(m, N)}\left(\theta, u_{h}\right)(i=1, \ldots, n)$ is given component-wise by

$\left[f_{i}^{(m, N)}\left(\theta, u_{h}\right)\right]_{j}=\left[P_{N}^{(0)}(\theta)\right]_{i}+\left(t_{j}-1\right) L^{2} \int_{0}^{t_{j}} \Psi_{i}\left(u_{h}(s)\right) d s+L^{2} \int_{t_{j}}^{1}(s-1) \Psi_{i}\left(u_{h}(s)\right) d s$,

where $j=0, \ldots, m$.

\subsection{Existence and local uniqueness via radii polynomials}

Inspired by the work of $[11,35]$ we provide a procedure for constructing a set

$B$ centered at a numerically derived approximate solution $\left(\hat{\theta}, \hat{u}_{h}\right)$ of $F^{(m, N)}=0$ which contains a genuine zero of the infinite dimensional nonlinear operator $F$ given by (14), and hence a symmetric homoclinic orbit of (1).

Consider the Banach space $X \stackrel{\text { def }}{=} \mathbb{R}^{n} \times C[0,1]^{n}$. Define

$$
X_{m} \stackrel{\text { def }}{=} \mathbb{R}^{n} \times\left(S_{h}\right)^{n} \cong \mathbb{R}^{n(m+2)} \quad \text { and } \quad X_{\infty} \stackrel{\text { def }}{=}\{0\}^{n} \times\left(\left(I-\Pi_{h}\right) C[0,1]\right)^{n},
$$

where we have used the decomposition (16). The projections $\Pi_{m}: X \rightarrow X_{m}$ and $\Pi_{\infty}: X \rightarrow X_{\infty}$ are given by

$$
\Pi_{m}(\theta, u)=\left(\begin{array}{c}
\theta \\
\left(\Pi_{h}\right)^{n} u
\end{array}\right) \quad \text { and } \quad \Pi_{\infty}(\theta, u)=\left(\begin{array}{c}
0 \\
\left(I-\Pi_{h}\right)^{n} u
\end{array}\right) .
$$


It then follows from (16) that $X$ decomposes as a direct sum of the form

$$
X=\Pi_{m} X \oplus \Pi_{\infty} X=X_{m} \oplus X_{\infty} .
$$

Recalling (14), one has

$$
\Pi_{m} F(\theta, u)=\left(\begin{array}{c}
P^{(1)}(\theta)-L^{2} \int_{0}^{1} \Psi(u(s)) d s \\
\left(\Pi_{h}\right)^{n}(f(\theta, u)-u)
\end{array}\right)
$$

and

$$
\Pi_{\infty} F(\theta, u)=\left(\begin{array}{c}
0 \\
\left(I-\Pi_{h}\right)^{n}(f(\theta, u)-u)
\end{array}\right) .
$$

Hence, we can write $F=\Pi_{m} F \oplus \Pi_{\infty} F$. In order to construct the fixed point equation, we assume that numerical calculations provide us with the following:

$\mathcal{A} .1$ Suppose we have an approximate solution $\left(\hat{\theta}, \hat{u}_{h}\right)$ of $F^{(m, N)}(\theta, u)=0$.

$\mathcal{A}$.2 Assume that we computed the Frechet derivative $D F^{(m, N)}\left(\hat{\theta}, \hat{u}_{h}\right)$.

$\mathcal{A} .3$ Assume that we computed an approximate inverse $A_{m}^{\dagger}$ of $D F^{(m, N)}\left(\hat{\theta}, \hat{u}_{h}\right)$.

$\mathcal{A}$.4 Suppose that $A_{m}^{\dagger}$ is injective.

In practice, showing that $\left\|I_{m}-A_{m}^{\dagger} D F^{(m, N)}\left(\hat{\theta}, \hat{u}_{h}\right)\right\|_{\infty}$ is strictly less than one is sufficient to prove that $A_{m}^{\dagger}$ is injective.

Let us define $T: X \rightarrow X$ by

$$
T(\theta, u) \stackrel{\text { def }}{=}\left(\Pi_{m}-A_{m}^{\dagger} \Pi_{m} F\right)(\theta, u)+\Pi_{\infty}(F(\theta, u)+(\theta, u)) .
$$

The next result follows immediately by the assumption $\mathcal{A} .4$ and by projecting $T$ and $F$ onto $X_{m}$ and $X_{\infty}$.

Lemma 2.4. One has that $T(\theta, u)=(\theta, u)$ if and only if $F(\theta, u)=0$.

In what follows we focus on studying the fixed points of $T$ rather than the zeros of $F$. To prove the existence of fixed points of $T$, we use a contraction argument. The maximum norm in $X_{m}$ is given by

$$
\left\|\Pi_{m}(\theta, u)\right\|_{X_{m}}=\max \left\{\|\theta\|_{\infty},\left\|\Pi_{h} u_{1}\right\|_{\infty}, \ldots,\left\|\Pi_{h} u_{n}\right\|_{\infty}\right\},
$$

and the norm in $X_{\infty}$ is given by

$$
\left\|\Pi_{\infty}(\theta, u)\right\|_{X_{\infty}}=\max _{i=1, \ldots, n}\left\{\left\|\left(I-\Pi_{h}\right) u_{i}\right\|_{\infty}\right\}=\max _{i=1, \ldots, n}\left\{\sup _{t \in[0,1]}\left\{\left|\left(I-\Pi_{h}\right) u_{i}(t)\right|\right\}\right\} .
$$

Observe that $\left(X_{m},\|\cdot\|_{X_{m}}\right)$ and $\left(X_{\infty},\|\cdot\|_{X_{\infty}}\right)$ are Banach spaces. Let us now consider the following set centered at $0 \in X$ :

$$
B(r, \omega) \stackrel{\text { def }}{=}\left\{(\theta, u) \mid\left\|\Pi_{m}(\theta, u)\right\|_{X_{m}} \leq r \text { and }\left\|\Pi_{\infty}(\theta, u)\right\|_{X_{\infty}} \leq \omega r\right\},
$$


where $\omega$ is a constant to be chosen later. The philosophy is that $r$ defines a radius of the box $B(r, \omega)$, while $\omega$ is a weight which can be used to adapt the radius of the tail. In what follows we work with a fixed $\omega$, whereas we treat $r$ as a variable.

Our goal is to use the contraction mapping theorem to prove the existence of a unique fixed point of $T$ within the set

$$
B \stackrel{\text { def }}{=}\left(\hat{\theta}, \hat{u}_{h}\right)+B(r, \omega) .
$$

This requires bounds on both the image and contractivity of $T$. This is incapsulated in the radii polynomials which are defined in terms of the following quantities.

Let the "deficit" function be

$$
y \stackrel{\text { def }}{=} T\left(\hat{\theta}, \hat{u}_{h}\right)-\left(\hat{\theta}, \hat{u}_{h}\right)=-A_{m}^{\dagger} \Pi_{m} F\left(\hat{\theta}, \hat{u}_{h}\right)+\Pi_{\infty} F\left(\hat{\theta}, \hat{u}_{h}\right),
$$

and suppose (see Section 2.4.1) we have constants $Y \stackrel{\text { def }}{=}\left(Y_{1}, \ldots, Y_{n(m+2)}, Y_{\infty}\right)$ such that the following estimates hold:

$$
\begin{aligned}
\left|\left(\Pi_{m} y\right)_{k}\right| & =\left|\left(-A_{m}^{\dagger} \Pi_{m} F\left(\hat{\theta}, \hat{u}_{h}\right)\right)_{k}\right| \leq Y_{k}, \quad k=1, \ldots, n(m+2), \\
\left\|\Pi_{\infty} y\right\|_{X_{\infty}} & =\left\|\Pi_{\infty} F\left(\hat{\theta}, \hat{u}_{h}\right)\right\|_{X_{\infty}} \leq Y_{\infty} .
\end{aligned}
$$

Moreover, let $w_{1}, w_{2} \in B(r, \omega)$ and $\hat{w} \stackrel{\text { def }}{=}\left(\hat{\theta}, \hat{u}_{h}\right)$. Define

$$
z=z\left(w_{1}, w_{2}\right) \stackrel{\text { def }}{=} D T\left(\hat{w}+w_{1}\right) w_{2}
$$

and suppose (see Section 2.4.2) that we have polynomial functions $Z(r) \stackrel{\text { def }}{=}$ $\left(Z_{1}(r), \ldots, Z_{n(m+2)}(r), Z_{\infty}(r)\right)$ with nonnegative coefficients such that the following estimates hold:

$$
\begin{aligned}
& \sup _{w_{1}, w_{2} \in B(r, \omega)}\left|\left(\Pi_{m} z\left(w_{1}, w_{2}\right)\right)_{k}\right| \leq Z_{k}, \quad k=1, \ldots, n(m+2), \\
& \sup _{w_{1}, w_{2} \in B(r, \omega)}\left\|\Pi_{\infty} z\left(w_{1}, w_{2}\right)\right\|_{X_{\infty}} \leq Z_{\infty} .
\end{aligned}
$$

The philosophy behind the construction of the radii polynomials is that each components of the bound $Z$ can be expanded as a polynomial of finite degree in the variable radius $r$ (see Section 2.4.2). This is an implicit assumption in the following definition.

Definition 2.5. The radii polynomials are given by

$$
\begin{aligned}
p_{k}(r) & \stackrel{\text { def }}{=} Y_{k}+Z_{k}(r)-r, \quad k=1, \ldots, n(m+2), \\
p_{n(m+2)+1}(r) & \stackrel{\text { def }}{=} Y_{\infty}+Z_{\infty}(r)-\omega r .
\end{aligned}
$$

The following result is a minor modification of Theorem 2.1 in [35] and Theorem 3.4 in [11]. It is a result based on a verification of the hypotheses of the Banach Fixed Point Theorem for $T$ working on $B$.

Theorem 2.6. If there exists an $r>0$ such that $p_{k}(r)<0$ for all $k=$ $1, \ldots, n(m+2)+1$, then there exists a unique fixed point of $T$, and hence a unique zero of $F$, within the set $B=\left(\hat{\theta}, \hat{u}_{h}\right)+B(r, \omega)$. 


\subsection{Explicit construction of the radii polynomials}

In this section, we provide an explicit construction of the bound $Y$ satisfying (22) and (23) and the bound $Z$ satisfying (25) and (26). Note that the final evaluation of the bounds $Y$ and $Z$ is a combination of analytic estimates and rigorous computations using interval arithmetic.

To estimate the terms

$$
P^{(j)}(\theta)=\sum_{|\alpha| \geq 0} a_{\alpha}^{(j)} \theta^{\alpha}, \quad j=0,1,
$$

which parametrize the stable manifold, we assume that we have computed exactly (using interval arithmetic) the coefficients $a_{\alpha}^{(j)}$ for $0 \leq|\alpha| \leq N$, forming the $N$-th order polynomial approximations

$$
P_{N}^{(j)}(\theta)=\sum_{0 \leq|\alpha| \leq N} a_{\alpha}^{(j)} \theta^{\alpha}
$$

Furthermore, we assume (see Section 4) that we have estimates of the form

$$
\sup _{|\theta|<\nu}\left|P^{(j)}(\theta)-P_{N}^{(j)}(\theta)\right|_{k} \leq \delta \quad \text { for } k=1, \ldots, n,
$$

for some $\delta>0$ and $\nu>0$, and

$$
\left|a_{\alpha}^{(j)}\right| \leq \frac{\delta}{\nu^{|\alpha|}} \quad \text { for all }|\alpha|>N, \text { and } j=0,1 .
$$

Finally, we assume that

$$
\max _{1 \leq j \leq n}\left|\hat{\theta}_{j}\right|<\nu
$$

\subsubsection{The bound $Y$}

One can use the splitting

$$
\Pi_{m} F\left(\hat{\theta}, \hat{u}_{h}\right)=F^{(m, N)}\left(\hat{\theta}, \hat{u}_{h}\right)+\left(\begin{array}{c}
P^{(1)}(\hat{\theta})-P_{N}^{(1)}(\hat{\theta}) \\
P^{(0)}(\hat{\theta})-P_{N}^{(0)}(\hat{\theta}) \\
\vdots \\
P^{(0)}(\hat{\theta})-P_{N}^{(0)}(\hat{\theta})
\end{array}\right),
$$

where $F^{(m, N)}$ is given by $(17)$ and where $P^{(0)}(\hat{\theta})-P_{N}^{(0)}(\hat{\theta}) \in \mathbb{R}^{n}$ appears $(m+1)$ times. Using interval arithmetic, one can evaluate $F^{(m, N)}\left(\hat{\theta}, \hat{u}_{h}\right)$, which should be small by the assumption $\mathcal{A} .1$, as well as compute an approximation $A_{m}^{\dagger}$ of the inverse of $D \Pi_{m} F\left(\hat{\theta}, \hat{u}_{h}\right)$. Under the assumption that $|\hat{\theta}|<\nu$ (which is easily checked), one can then use (29) to choose

$$
\left\{Y_{i}\right\}_{i=1, \ldots, n(m+2)} \geq\left|A_{m}^{\dagger} F^{(m, N)}\left(\hat{\theta}, \hat{u}_{h}\right)\right|+\delta\left|A_{m}^{\dagger}\right| \mathbf{1},
$$


where $\mathbf{1} \stackrel{\text { def }}{=}(1,1, \cdots, 1) \in \mathbb{R}^{n(m+2)}$, and the absolute values are to be interpreted component-wise. Computing the right-hand side of (31) using interval arithmetic, we find explicit bounds $Y_{i}, i=1, \ldots, n(m+2)$ such that the estimates (22) are satisfied.

The following result provides a way to compute the bound $Y_{\infty}$ in (23).

Lemma 2.7. Let $Y_{\infty}$ be such that

$$
Y_{\infty} \geq \max _{i=1, \ldots, n}\left\{\max _{k=0, \ldots, m-1}\left\{\frac{L^{2}}{8}\left(t_{k+1}-t_{k}\right)^{2} \sup _{s \in\left[t_{k}, t_{k+1}\right]}\left|\Psi_{i}\left(\hat{u}_{h}(s)\right)\right|\right\}\right\} .
$$

Then,

$$
\left\|\Pi_{\infty} y\right\|_{X_{\infty}} \leq Y_{\infty}
$$

Proof. By definition

$$
\left\|\Pi_{\infty} y\right\|_{X_{\infty}}=\left\|\Pi_{\infty} F\left(\hat{\theta}, \hat{u}_{h}\right)\right\|_{X_{\infty}}=\max _{i=1, \ldots, n}\left\{\left\|\left(I-\Pi_{h}\right) f_{i}\left(\hat{\theta}, \hat{u}_{h}\right)\right\|_{\infty}\right\} .
$$

Observe that $f_{i}\left(\hat{\theta}, \hat{u}_{h}\right) \in C^{2}[0,1]$, for $i=1, \ldots, n$. Using [32, Theorem 2.6], (13) and

$$
\frac{d^{2}}{d t^{2}} f(\theta, u)=L^{2} \Psi(u)
$$

one obtains

$$
\begin{aligned}
\left\|\left(I-\Pi_{h}\right) f_{i}\left(\hat{\theta}, \hat{u}_{h}\right)\right\|_{\infty} & \leq \max _{k=0, \ldots, m-1}\left\{\frac{\left(t_{k+1}-t_{k}\right)^{2}}{8} \sup _{s \in\left[t_{k}, t_{k+1}\right]}\left|\frac{d^{2}}{d^{2} t} f_{i}\left(\hat{\theta}, \hat{u}_{h}\right)(s)\right|\right\} \\
& =\max _{k=0, \ldots, m-1}\left\{\frac{L^{2}}{8}\left(t_{k+1}-t_{k}\right)^{2} \sup _{s \in\left[t_{k}, t_{k+1}\right]}\left|\Psi_{i}\left(\hat{u}_{h}(s)\right)\right|\right\} .
\end{aligned}
$$

For the application to the Gray-Scott system presented in Section 5, we estimate the right-hand side of (32) by doing a very crude interval arithmetic calculation of $\Psi_{i}\left(\hat{u}_{h}\right) \in \Psi_{i}\left(\left[\hat{u}_{k}, \hat{u}_{k+1}\right]\right)$ on each interval $\left[t_{k}, t_{k+1}\right]$.

\subsubsection{The bound $Z$ as a polynomial in $r$}

Commutativity of the differential operator and linear operators $\left(\Pi_{m}\right.$ and $\left.A_{m}^{\dagger}\right)$ implies that

$$
\begin{aligned}
\Pi_{m} z\left(w_{1}, w_{2}\right)= & \Pi_{m}\left(D T\left(\hat{w}+w_{1}\right) w_{2}\right) \\
= & D \Pi_{m}\left(\hat{w}+w_{1}\right) w_{2}-A_{m}^{\dagger} D \Pi_{m} F\left(\hat{w}+w_{1}\right) w_{2} \\
= & {\left[I_{m}-A_{m}^{\dagger} D \Pi_{m} F(\hat{w})\right] \Pi_{m} w_{2} } \\
& \quad-A_{m}^{\dagger}\left[D \Pi_{m} F\left(\hat{w}+w_{1}\right) w_{2}-D \Pi_{m} F(\hat{w}) \Pi_{m} w_{2}\right],
\end{aligned}
$$


where the factor $I_{m}-A_{m}^{\dagger} D \Pi_{m} F(\hat{w})$ is expected to be small due to assumption $\mathcal{A} .3$.

Let us now expand $\Pi_{m} z$ as polynomials in the variable radius $r>0$. Considering $\tilde{w}_{1}, \tilde{w}_{2} \in B(1, \omega)$ so that $w_{1}=r \tilde{w}_{1}$ and $w_{2}=r \tilde{w}_{2}$, one obtains that

$$
\begin{aligned}
\Pi_{m} z\left(w_{1}, w_{2}\right)=[ & \left.\left(I_{m}-A_{m}^{\dagger} D \Pi_{m} F(\hat{w})\right) \Pi_{m} \tilde{w}_{2}\right] r \\
& \quad-A_{m}^{\dagger}\left[D \Pi_{m} F\left(\hat{w}+\tilde{w}_{1} r\right) \tilde{w}_{2}-D \Pi_{m} F(\hat{w}) \Pi_{m} \tilde{w}_{2}\right] r \\
=[ & \left.\left(I_{m}-A_{m}^{\dagger} D \Pi_{m} F(\hat{w})\right) \Pi_{m} \tilde{w}_{2}\right] r-A_{m}^{\dagger} \eta^{\prime}(0) r,
\end{aligned}
$$

where

$$
\eta(\tau) \stackrel{\text { def }}{=} \Pi_{m} F\left(\hat{w}+\tilde{w}_{1} r+\tau \tilde{w}_{2}\right)-\Pi_{m} F\left(\hat{w}+\tau \Pi_{m} \tilde{w}_{2}\right) .
$$

The next step is to expand $\eta^{\prime}(0)$ in terms of the variable radius $r$. Let $\hat{w}=\left(\hat{\theta}, \hat{u}_{h}\right)$ and write $\tilde{w}_{1}=(\tilde{\vartheta}, \tilde{u})$ and $\tilde{w}_{2}=(\tilde{\varphi}, \tilde{v})$. We start by considering the first $n$ entries of $\eta$, corresponding to the first component of $\Pi_{m} F$ given by (18). One has

$$
\begin{aligned}
\left\{\eta_{k}(\tau)\right\}_{k=1, \ldots, n}=\sum_{|\alpha| \geq 1} & a_{\alpha}^{(1)}\left[(\hat{\theta}+\tilde{\vartheta} r+\tau \tilde{\varphi})^{\alpha}-(\hat{\theta}+\tau \tilde{\varphi})^{\alpha}\right] \\
& -L^{2} \int_{0}^{1}\left[\Psi\left(\hat{u}_{h}+\tilde{u} r+\tau \tilde{v}\right)-\Psi\left(\hat{u}_{h}+\tau\left(\Pi_{h}\right)^{n} \tilde{v}\right)\right] d s,
\end{aligned}
$$

and hence, denoting by $e_{i}$ the $i$-th unit vector,

$$
\begin{aligned}
\left\{\eta_{k}^{\prime}(0)\right\}_{k=1, \ldots, n}=\sum_{|\alpha| \geq 1} a_{\alpha}^{(1)} & \left(\sum_{\substack{i=1 \\
\left(\alpha_{i}>0\right)}}^{n} \alpha_{i} \tilde{\varphi}_{i}\left[(\hat{\theta}+\tilde{\vartheta} r)^{\alpha-e_{i}}-\hat{\theta}^{\alpha-e_{i}}\right]\right) \\
& -L^{2} \int_{0}^{1}\left[D \Psi\left(\hat{u}_{h}+\tilde{u} r\right) \tilde{v}-D \Psi\left(\hat{u}_{h}\right)\left(\Pi_{h}\right)^{n} \tilde{v}\right] d s .
\end{aligned}
$$

We proceed by estimating the two terms in the right-hand side separately.

For the first term we have, for each component $k=1, \ldots, n$,

$$
\begin{aligned}
& \left\{\sum_{|\alpha| \geq 1} a_{\alpha}^{(1)}\left(\sum_{\substack{i=1 \\
\left(\alpha_{i}>0\right)}}^{n} \alpha_{i} \tilde{\varphi}_{i}\left[\left(\hat{\theta}+\tilde{\vartheta}_{r}\right)^{\alpha-e_{i}}-\hat{\theta}^{\alpha-e_{i}}\right]\right)\right\}_{k} \\
& =\left\{\sum_{|\alpha| \geq 1} a_{\alpha}^{(1)}\left(\sum_{\substack{i=1 \\
\left(\alpha_{i}>0\right)}}^{n} \alpha_{i} \tilde{\varphi}_{i} \sum_{\substack{l=1 \\
\left(\alpha_{l}>\delta_{i, l}\right)}}^{n}\left(\alpha_{l}-\delta_{i, l}\right) \tilde{\vartheta}_{l}(\hat{\theta}+\xi \tilde{\vartheta} r)^{\alpha-e_{i}-e_{l}}\right) r\right\}_{k} \\
& =\sum_{i=1}^{n} \sum_{l=1}^{n}\left\{\sum_{\alpha \in \mathcal{S}_{i, l}} a_{\alpha}^{(1)} \alpha_{i}\left(\alpha_{l}-\delta_{i, l}\right) \tilde{\varphi}_{i} \tilde{\vartheta}_{l}(\hat{\theta}+\xi \tilde{\vartheta} r)^{\alpha-e_{i}-e_{l}}\right\}_{k}
\end{aligned}
$$


for some $\xi=\xi(k, i, l, \alpha) \in[0,1]$, where $\delta_{i, l}$ is the Kronecker's symbol, and, for $i, l \in\{1, \ldots, n\}$,

$$
\mathcal{S}_{i, l} \stackrel{\text { def }}{=}\left\{\alpha=\left(\alpha_{1}, \ldots, \alpha_{n}\right) \in \mathbb{N}^{n} \mid \alpha_{i}>0 \text { and } \alpha_{l}>\delta_{i, l}\right\} .
$$

We now choose an a priori bound $r \leq r_{*}$, where we fix, see (30),

$$
0<r_{*}<\nu-\max _{1 \leq j \leq n}\left|\hat{\theta}_{j}\right| .
$$

Then, using $\left|\tilde{\varphi}_{i}\right|,\left|\tilde{\vartheta}_{l}\right| \leq 1$ for all $i, l \in\{1, \ldots, n\}$, we obtain the component-wise bound

$$
\left|\sum_{\alpha \in \mathcal{S}_{i, l}} a_{\alpha}^{(1)} \alpha_{i}\left(\alpha_{l}-\delta_{i, l}\right) \tilde{\varphi}_{i} \tilde{\vartheta}_{l}(\hat{\theta}+\xi \tilde{\vartheta} r)^{\alpha-e_{i}-e_{l}}\right| \leq\left|\sum_{\alpha \in \mathcal{S}_{i, l}} a_{\alpha}^{(1)} \alpha_{i}\left(\alpha_{l}-\delta_{i, l}\right) \sigma^{\alpha-e_{i}-e_{l}}\right|,
$$

where $\sigma=\left(\sigma_{1}, \ldots, \sigma_{n}\right)$, with $\sigma_{j}=\sigma_{j}(i, l, \alpha) \in\left[\hat{\theta}_{j}-r_{*}, \hat{\theta}_{j}+r_{*}\right]$ for $j \in\{1, \ldots, n\}$.

With this in mind we estimate

$$
\sum_{i=1}^{n} \sum_{l=1}^{n}\left|\sum_{\alpha \in \mathcal{S}_{i, l}} a_{\alpha}^{(1)} \alpha_{i}\left(\alpha_{l}-\delta_{i, l}\right) \sigma^{\alpha-e_{i}-e_{l}}\right| \leq \Lambda^{(1)}, \quad \text { for all } \sigma_{j} \in\left[\hat{\theta}_{j}-r_{*}, \hat{\theta}_{j}+r_{*}\right] \text {, }
$$

with bound $\Lambda^{(1)} \in \mathbb{R}_{+}^{n}$. The computation of the bound $\Lambda^{(1)}$, under the assumption that $\left|\sigma_{j}\right|<\nu$ for $j=1, \ldots, n$, is a combination of computations and analysis, see Section 5.2.1 for more details and the explicit construction for the Gray-Scott system.

To estimate the second term in the right-hand side of (36) we can write, for polynomial $\Psi$,

$$
D \Psi\left(\hat{u}_{h}+\tilde{u} r\right) \tilde{v}-D \Psi\left(\hat{u}_{h}\right)\left(\Pi_{h}\right)^{n} \tilde{v}=\sum_{\ell=1}^{d} \mathrm{v}^{(\ell)} r^{\ell-1},
$$

for some $d \in \mathbb{N}$ and a set of vector functions $\mathrm{v}^{(\ell)}=\mathrm{v}^{(\ell)}\left(\hat{u}_{h}, \tilde{u}, \tilde{v}\right) \in \mathbb{R}^{n}, \ell=$ $1, \ldots, d$. For an example of how to determine the functions $\mathrm{v}^{(\ell)}$, see Section 5.2.2 in the context of the Gray-Scott equation. There it is also explained how to use the fact that $|\tilde{u}(s)| \leq 1$ and $|\tilde{v}(s)| \leq 1$ to obtain estimates of the form

$$
\int_{0}^{1}\left|\mathrm{v}^{(\ell)}(s)\right| d s \leq \Gamma^{(\ell)}, \quad \text { for all }\|\tilde{u}\|_{\infty},\|\tilde{v}\|_{\infty} \leq 1,
$$

with $\Gamma^{(\ell)} \in \mathbb{R}_{+}^{n}$ for $\ell=1, \ldots, d$. Combining the bounds (37) and (39), one can finally obtain the following upper bound for (36)

$$
\left|\left\{\eta_{k}^{\prime}(0)\right\}_{k=1, \ldots, n}\right| \leq \Lambda^{(1)} r+L^{2} \sum_{\ell=1}^{d} \Gamma^{(\ell)} r^{\ell-1}
$$


Now that we have found an upper bound for the first $n$ components of $\eta^{\prime}(0)$, let us do the same for its remaining $n(m+1)$ components. Note that these components of $\eta$ correspond to the second component of $\Pi_{m} F$ given by (18). First, let us recall (35) and fix a mesh index $j \in\{0, \ldots, m\}$. Then, evaluating $\eta$ at the mesh point $t_{j}$ gives the $n$ dimensional vector

$$
\begin{aligned}
& \left\{\eta_{k}(\tau)\right\}_{k=n(j+1), \ldots, n(j+2)}= \\
& \sum_{|\alpha| \geq 1} a_{\alpha}^{(0)}\left[(\hat{\theta}+\tilde{\vartheta} r+\tau \tilde{\varphi})^{\alpha}-(\hat{\theta}+\tau \tilde{\varphi})^{\alpha}\right]-\left(\Pi_{h}\right)^{n} \tilde{u} r \\
& \quad+\left(t_{j}-1\right) L^{2} \int_{0}^{t_{j}}\left[\Psi\left(\hat{u}_{h}+\tilde{u} r+\tau \tilde{v}\right)-\Psi\left(\hat{u}_{h}+\tau\left(\Pi_{h}\right)^{n} \tilde{v}\right)\right] d s \\
& \quad+L^{2} \int_{t_{j}}^{1}(s-1)\left[\Psi\left(\hat{u}_{h}+\tilde{u} r+\tau \tilde{v}\right)-\Psi\left(\hat{u}_{h}+\tau\left(\Pi_{h}\right)^{n} \tilde{v}\right)\right] d s
\end{aligned}
$$

As in the case for the bound $\Lambda^{(1)}$ given by (37), one can consider a bound $\Lambda^{(0)} \in \mathbb{R}_{+}^{n}$ such that

$$
\sum_{i=1}^{n} \sum_{l=1}^{n}\left|\sum_{\alpha \in \mathcal{S}_{i, l}} a_{\alpha}^{(0)} \alpha_{i}\left(\alpha_{l}-\delta_{i, l}\right) \sigma^{\alpha-e_{i}-e_{l}}\right| \leq \Lambda^{(0)},
$$

for all $\sigma_{k}=\sigma_{k}(i, l, \alpha) \in\left[\hat{\theta}_{k}-r_{*}, \hat{\theta}_{k}+r_{*}\right], k=1, \ldots, n$. Combining the expansion (38) and the bound (41) allow us to obtain, for any fixed $j \in\{0, \ldots, m\}$,

$$
\left|\left\{\eta_{k}^{\prime}(0)\right\}_{k=n(j+1), \ldots, n(j+2)}\right| \leq \Lambda^{(0)} r+L^{2} \sum_{\ell=1}^{d}\left[\left(1-t_{j}\right) \Gamma_{j}^{(\ell)}+\widetilde{\Gamma}_{j}^{(\ell)}\right] r^{\ell-1},
$$

where $\Gamma_{j}^{(\ell)}$ and $\widetilde{\Gamma}_{j}^{(\ell)}$ are bounds

$$
\begin{array}{r}
\int_{0}^{t_{j}}\left|\mathrm{v}^{(\ell)}(s)\right| d s \leq \Gamma_{j}^{(\ell)}, \\
\int_{t_{j}}^{1}(1-s)\left|\mathrm{v}^{(\ell)}(s)\right| d s \leq \widetilde{\Gamma}_{j}^{(\ell)},
\end{array}
$$

for all $\|\tilde{u}\|_{\infty},\|\tilde{v}\|_{\infty} \leq 1$.

We now have component-wise upper bounds for $\left|\eta^{\prime}(0)\right|$, which are given by (40) and (42), which we summarize as

$$
\left|\eta^{\prime}(0)\right| \leq \sum_{\ell=1}^{d} \mathrm{~V}^{(\ell)} r^{\ell-1}
$$

where $\mathrm{V}^{(1)}, \ldots, \mathrm{V}^{(d)} \in \mathbb{R}_{+}^{n(m+2)}$.

Applying bound (45) to equation (34) provides the estimate

$$
\begin{aligned}
\left|\Pi_{m} z\left(w_{1}, w_{2}\right)\right| & =\left|\left[\left(I_{m}-A_{m}^{\dagger} D \Pi_{m} F(\hat{w})\right) \Pi_{m} \tilde{w}_{2}\right] r-A_{m}^{\dagger} \eta^{\prime}(0) r\right| \\
& \leq\left(\left|I_{m}-A_{m}^{\dagger} D \Pi_{m} F\left(\hat{\theta}, \hat{u}_{h}\right)\right| \mathbf{1}\right) r+\sum_{\ell=1}^{d}\left|A_{m}^{\dagger}\right| \mathrm{V}^{(\ell)} r^{\ell},
\end{aligned}
$$


where here $\mathbf{1}=(1,1, \ldots, 1) \in \mathbb{R}^{n(m+2)}$. For $\ell=1, \ldots, d$, define

$$
\begin{aligned}
& Z^{(1)} \stackrel{\text { def }}{=}\left|I_{m}-A_{m}^{\dagger} D \Pi_{m} F\left(\hat{\theta}, \hat{u}_{h}\right)\right| \mathbf{1}+\left|A_{m}^{\dagger}\right| \mathrm{V}^{(1)}, \\
& Z^{(\ell)} \stackrel{\text { def }}{=}\left|A_{m}^{\dagger}\right| \mathrm{V}^{(\ell)}, \quad \ell=2, \ldots, d .
\end{aligned}
$$

Hence

$$
\sup _{w_{1}, w_{2} \in B(r, \omega)}\left|\Pi_{m} z\left(w_{1}, w_{2}\right)\right| \leq \sum_{\ell=1}^{d} Z^{(\ell)} r^{\ell} .
$$

Finally, define $Z_{1}, \ldots, Z_{n(m+2)}$ by

$$
Z_{k}(r) \stackrel{\text { def }}{=} \sum_{\ell=1}^{d} Z_{k}^{(\ell)} r^{\ell}, \quad k=1, \ldots, n(m+2) .
$$

For the remaining bound (26) we consider

$$
\Pi_{\infty} z\left(w_{1}, w_{2}\right)=\Pi_{\infty}\left(D T\left(\hat{w}+w_{1}\right) w_{2}\right)=D \Pi_{\infty}(F+I)\left(\hat{w}+w_{1}\right) w_{2} .
$$

For polynomial $\Psi$ we can write, cf. (38),

$$
D \Psi_{i}\left(\hat{u}_{h}+\tilde{u} r\right) \tilde{v}=\sum_{\ell=1}^{d} \overline{\mathrm{v}}_{i}^{(\ell)} r^{\ell-1} .
$$

As before, using $|\tilde{u}(s)| \leq 1$ and $|\tilde{v}(s)| \leq 1$, we can derive bounds

$$
\sup _{s \in\left[t_{k}, t_{k+1}\right]}\left|\overline{\mathrm{v}}_{i}^{(\ell)}(s)\right| \leq \bar{\Gamma}_{i, k}^{(\ell)}, \quad \text { for all }\|\tilde{u}\|_{\infty},\|\tilde{v}\|_{\infty} \leq 1,
$$

for $k=1, \ldots, m-1, i=1, \ldots, n, \ell=1, \ldots, d$, see Section 5.2.2. The following result provides an upper bound for the $X_{\infty}$ norm of (47) in terms of a polynomial in the variable radius $r>0$.

Lemma 2.8. Define

$$
Z_{\infty}(r) \stackrel{\text { def }}{=} \sum_{\ell=1}^{d} Z_{\infty}^{(\ell)} r^{\ell},
$$

where $Z_{\infty}^{(1)}, \ldots, Z_{\infty}^{(d)}$ are bounds such that

$$
\max _{i=1, \ldots, n}\left\{\max _{k=0, \ldots, m-1}\left\{\frac{L^{2}}{8}\left(t_{k+1}-t_{k}\right)^{2} \bar{\Gamma}_{i, k}^{(\ell)}\right\}\right\} r \leq \sum_{\ell=1}^{d} Z_{\infty}^{(\ell)} r^{\ell} .
$$

Then

$$
\sup _{w_{1}, w_{2} \in B(r, \omega)}\left\|\Pi_{\infty} z\left(w_{1}, w_{2}\right)\right\|_{X_{\infty}} \leq Z_{\infty}(r) .
$$


Proof. From [32, Theorem 2.6] one finds

$$
\begin{aligned}
\| \Pi_{\infty} & z\left(w_{1}, w_{2}\right) \|_{X_{\infty}} \\
& =\left\|D \Pi_{\infty}(F+I)\left(\hat{w}+w_{1}\right) w_{2}\right\|_{X_{\infty}} \\
& =\max _{i=1, \ldots, n}\left\{\left\|\left(I-\Pi_{h}\right) D f_{i}\left(\hat{w}+w_{1}\right) w_{2}\right\|_{\infty}\right\} \\
& \leq \max _{i=1, \ldots, n}\left\{\max _{k=0, \ldots, m-1}\left\{\frac{\left(t_{k+1}-t_{k}\right)^{2}}{8} \sup _{s \in\left[t_{k}, t_{k+1}\right]}\left|\frac{d^{2}}{d^{2} t}\left[D f_{i}\left(\hat{w}+w_{1}\right) w_{2}\right](s)\right|\right\}\right\} \\
& =\max _{i=1, \ldots, n}\left\{\max _{k=0, \ldots, m-1}\left\{\frac{L^{2}}{8}\left(t_{k+1}-t_{k}\right)^{2} \sup _{s \in\left[t_{k}, t_{k+1}\right]}\left|D \Psi_{i}\left(\hat{u}_{h}+\tilde{u} r\right) \tilde{v}\right|\right\}\right\} r .
\end{aligned}
$$

The assertion now follows from (48) and (49).

\section{Parameterization of the invariant manifolds}

This section provides a review of the parameterization method for invariant manifolds as developed in $[7,8,9]$, adapted to our current setting.

A critical ingredient of the parameterization method is that once the local approximation is computed, it is possible to efficiently validate, a-posteriori, the numerical results. Validation is discussed in detail in section 4 as well as in [9]. Because we are interested in numerical computations, we develop explicit formula for the necessary constants appearing in all estimates. The estimates are computationally convenient, rather than theoretically sharp.

\subsection{Background: analytic functions and $N$-tails}

We endow $\mathbb{C}^{K}$ with the supremum norm

$$
|z|=\left|\left(z_{1}, \ldots, z_{K}\right)\right|=\max _{1 \leq i \leq K}\left|z_{i}\right| .
$$

Under this norm the ball of radius $\nu$ centered at the origin in $\mathbb{C}^{K}$ is the polydisk

$$
\mathbb{B}_{\nu}=\left\{z \in \mathbb{C}^{K}|| z_{i} \mid<\nu \text { for } 1 \leq i \leq K\right\} .
$$

A bounded analytic function $g: \mathbb{B}_{\nu} \subset \mathbb{C}^{K_{1}} \rightarrow \mathbb{C}^{K_{2}}$ for which $g(0)=0$ has a power series expansion

$$
g(z)=\sum_{|\alpha| \geq 1} a_{\alpha} z^{\alpha}
$$

where $\alpha=\left(\alpha_{1}, \ldots, \alpha_{K_{1}}\right) \in \mathbb{N}^{K_{1}}$ denotes a $K_{1}$-dimensional multi-index, with $|\alpha|=\alpha_{1}+\ldots+\alpha_{K_{1}}$, so that $z^{\alpha}=z_{1}^{\alpha_{1}} \cdot \ldots \cdot z_{K_{1}}^{\alpha_{K_{1}}}$, and $a_{\alpha} \in \mathbb{C}^{K_{2}}$ are $K_{2^{-}}$ dimensional complex coefficients. When the coefficients $a_{\alpha}$ are real, we say that $g$ is real analytic. 
We employ two norms on the space of bounded analytic functions from $\mathbb{B}_{\nu} \subset \mathbb{C}^{K_{1}}$ into $\mathbb{C}^{K_{2}}$ : the supremum norm

$$
\|g\|_{\nu} \stackrel{\text { def }}{=} \sup _{\left|z_{i}\right| \leq \nu}\left|g\left(z_{1}, \ldots, z_{n}\right)\right|=\sup _{\left|z_{i}\right|=\nu}\left|g\left(z_{1}, \ldots, z_{n}\right)\right|,
$$

where the (second) equality is due to the maximum modulus principle [1], and the $\nu$-weighted $\Sigma$-norm

$$
\|g\|_{\Sigma, \nu} \equiv \sum_{|\alpha| \geq 1}\left|a_{\alpha}\right| \nu^{|\alpha|}
$$

The latter norm is inexpensive to compute numerically when $g$ is a polynomial, and we have the bound

$$
\|g\|_{\nu} \leq\|g\|_{\Sigma, \nu} .
$$

A $K_{2} \times K_{1}$ matrix $A$ with complex entries defines a linear operator from $\mathbb{C}^{K_{1}}$ to $\mathbb{C}^{K_{2}}$, and

$$
\|A\|_{M} \stackrel{\text { def }}{=} \sup _{|z|=1}|A z|
$$

where $|\cdot|$ is the norm defined by (52). If the entries of $A(z)$ are themselves bounded analytic functions on $\mathbb{B}_{\nu}$ then we define the norm

$$
\|A\|_{M, \nu} \stackrel{\text { def }}{=} \sup _{z \in \mathbb{B}_{\nu}}\|A(z)\|_{M} .
$$

For $g: \mathbb{B}_{\nu} \subset \mathbb{C}^{K_{1}} \rightarrow \mathbb{C}^{K_{2}}$ the non-constant matrix-vector product $A(z) \cdot g(z)$ defines an analytic function from $\mathbb{B}_{\nu} \subset \mathbb{C}^{K_{1}}$ into $\mathbb{C}^{K_{2}}$, and we have

$$
\|A \cdot g\|_{\nu} \leq\|A\|_{M, \nu}\|g\|_{\nu} .
$$

Definition 3.1. An analytic function $h: \mathbb{B}_{\nu} \subset \mathbb{C}^{K_{1}} \rightarrow \mathbb{C}^{K_{2}}$ is an analytic $N$-tail if

$$
h(0)=D h(0)=\ldots=D^{N} h(0)=0 .
$$

Let $\mathcal{X}$ denote the space of bounded analytic functions on $\mathbb{B}_{\nu} \subset \mathbb{C}^{K_{1}}$ mapping into $\mathbb{C}^{K_{2}}$, and $\mathcal{X}_{0}$ denote the analytic $N$-tails in $\mathcal{X}$. Throughout we suppress dependence of $\mathcal{X}$ and $\mathcal{X}_{0}$ on $\nu, K_{1}, K_{2}$ and even $N$, as these will always be clear from the context. The spaces $\mathcal{X}$ and $\mathcal{X}_{0}$ equipped with the supremum norm $\|\cdot\|_{\nu}$ are Banach spaces. The norm of a bounded linear operator $\mathfrak{L}: \mathcal{X} \rightarrow \mathcal{X}$ is given by

$$
\|\mathfrak{L}\|_{\mathcal{X}}=\sup _{x \in \mathcal{X},\|x\|_{\nu}=1}\|\mathcal{L} x\|_{\nu}
$$

The following estimate for analytic $N$-tails is elementary but essential in what follows.

Lemma 3.2 (rescaling for $N$-tails). Suppose that $h: \mathbb{B}_{\nu} \subset \mathbb{C}^{K_{1}} \rightarrow \mathbb{C}^{K_{2}}$ is an analytic $N$-tail, that $\|h\|_{\nu}=M<\infty$, and that $\beta$ is a complex scalar with $|\beta| \leq 1$. Then

$$
\|h \circ \beta\|_{\nu} \leq|\beta|^{N+1}|| h \|_{\nu} .
$$


Proof. Consider first the situation, in one complex variable, where $f: \mathbb{C} \rightarrow \mathbb{C}$ is analytic and bounded on the closed unit disk, $f(0)=f^{\prime}(0)=\ldots=f^{N}(0)=0$, and $|f(z)| \leq M$ for $|z| \leq 1$. Then

$$
f(z)=z^{N+1} g(z)
$$

for some function $g$, which is analytic on the closed unit disk. The maximum modulus principle implies that $g$ attains its maximum in the compact set $\overline{\mathbb{B}_{1}}$ on the boundary only, say at $z_{0}$ with $\left|z_{0}\right|=1$. Thus

$$
\left|\frac{f(z)}{z^{N+1}}\right|=|g(z)| \leq\left|g\left(z_{0}\right)\right|=\left|f\left(z_{0}\right)\right| \leq M,
$$

so that $|f(z)| \leq|z|^{N+1} M$.

Now suppose that $h: \mathbb{B}_{\nu} \subset \mathbb{C}^{K} \rightarrow \mathbb{C}$ is an analytic $N$-tail such that $\|h\|_{\nu}=$ $M$. Let $\beta$ be a complex number with $|\beta| \leq 1$. For fixed $z_{0} \in \mathbb{B}_{\nu}$ the function $f_{z_{0}}(\beta) \equiv h\left(\beta z_{0}\right)$ is an analytic function of one complex variable (namely the variable $\beta)$. In fact we have that $f_{z_{0}}(\beta)$ is defined on the closed unit disk $\overline{\mathbb{B}_{1}} \subset \mathbb{C}$. Furthermore, $\left|f_{z_{0}}(\beta)\right| \leq M$, and $f_{z_{0}}(0)=D f_{z_{0}}(0)=\ldots=D^{N} f_{z_{0}}(0)=0$ as $h$ is an $N$-tail. Thus, by the one dimensional argument,

$$
\left|f_{z_{0}}(\beta)\right| \leq|\beta|^{N+1} M,
$$

uniformly in $z_{0}$. Therefore

$$
\|h \circ \beta\|_{\nu}=\sup _{z \in \mathbb{B}_{\nu}}|h(\beta z)|=\sup _{z \in \mathbb{B}_{\nu}}\left|f_{z}(\beta)\right| \leq|\beta|^{N+1} M=|\beta|^{N+1}|| h \|_{\nu} .
$$

If $h: \mathbb{B}_{\nu} \subset \mathbb{C}^{K_{1}} \rightarrow \mathbb{C}^{K_{2}}$ then the result follows by applying the previous result component-wise.

The following result estimates the derivative of an analytic 2-tail $R$, a remainder term that appears in Section 4.2, on a polydisk of radius $\delta<\delta_{0}$, in terms of a bound for $R$ on a polydisk of radius $\delta_{0}$.

Lemma 3.3 (Cauchy bound for a second order zero). Suppose that $R: \mathbb{B}_{\delta_{0}} \subset$ $\mathbb{C}^{K} \rightarrow \mathbb{C}^{K}$ is an analytic 2-tail, that $\|R\|_{\delta_{0}}<\infty$. Then for all $\delta<\delta_{0} /(K+2)$ we have

$$
\|D R\|_{\delta} \leq \frac{K(K+2)^{K+2}}{(K+1)^{K+1}} \frac{\delta}{\delta_{0}^{2}}\|R\|_{\delta_{0}} .
$$

Proof. Begin by considering a function $f: \mathbb{B}_{\delta_{0}} \subset \mathbb{C}^{K} \rightarrow \mathbb{C}$ having $f(0)=$ $D f(0)=0$. Then for any $|z| \leq \delta$, and $1 \leq j \leq K$, the Cauchy integral formula gives

$$
\begin{aligned}
& \frac{\partial}{\partial z_{j}} f\left(z_{1}, \ldots, z_{K}\right) \\
& \quad=\frac{1}{(2 \pi i)^{K}} \int_{\left|\zeta_{1}\right|=\delta_{*}} \ldots \int_{\left|\zeta_{K}\right|=\delta_{*}} \frac{f\left(\zeta_{1}, \ldots, \zeta_{K}\right) d \zeta_{1} \ldots d \zeta_{K}}{\left(\zeta_{1}-z_{1}\right) \cdots\left(\zeta_{j}-z_{j}\right)^{2} \cdots\left(\zeta_{K}-z_{K}\right)},
\end{aligned}
$$


where $\delta<\delta_{*}<\delta_{0}$. Writing $\zeta_{k}=\delta_{*} e^{i \theta_{k}}$, we note that by Lemma 3.2

$$
f\left(\delta_{*} e^{i \theta_{1}}, \ldots, \delta_{*} e^{i \theta_{K}}\right)=f\left(\frac{\delta_{*}}{\delta_{0}} \delta_{0} e^{i \theta_{1}}, \ldots, \frac{\delta_{*}}{\delta_{0}} \delta_{0} e^{i \theta_{K}}\right) \leq\left(\delta_{*} / \delta_{0}\right)^{2}\|f\|_{\delta_{0}} .
$$

Hence, denoting $\delta_{*}=(1+\tau) \delta$ with $\tau>0$, we obtain the estimate

$$
\begin{aligned}
\mid \frac{\partial}{\partial z_{j}} & f(z) \mid \\
& =\left|\frac{1}{(2 \pi i)^{K}} \int_{0}^{2 \pi} \cdots \int_{0}^{2 \pi} \frac{f\left(\delta_{*} e^{i \theta_{1}}, \ldots, \delta_{*} e^{i \theta_{K}}\right)\left(i \delta_{*}\right)^{K} e^{i\left(\theta_{1}+\ldots+\theta_{K}\right)} d \theta_{1} \ldots d \theta_{K}}{\left(\delta_{*} e^{i \theta_{1}}-z_{1}\right) \cdots\left(\delta_{*} e^{i \theta_{j}}-z_{j}\right)^{2} \cdots\left(\delta_{*} e^{i \theta_{K}}-z_{K}\right)}\right| \\
& \leq \frac{1}{(2 \pi)^{K}} \int_{0}^{2 \pi} \cdots \int_{0}^{2 \pi} \frac{\left(\delta_{*} / \delta_{0}\right)^{2}\|f\|_{\delta_{0}} \delta_{*}^{K} d \theta_{1} \ldots d \theta_{L}}{\left|\delta_{*}-\delta\right| \cdots\left|\delta_{*}-\delta\right|^{2} \cdots\left|\delta_{*}-\delta\right|} \\
& \leq \frac{(1+\tau)^{K+2}}{\tau^{K+1}} \frac{\delta}{\delta_{0}^{2}}\|f\|_{\delta_{0}} .
\end{aligned}
$$

Since estimate (54) holds for all $0<\tau<\frac{\delta_{0}}{\delta}-1<K+1$, and because

$$
\inf _{\tau>0}\left(\frac{(1+\tau)^{K+2}}{\tau^{K+1}}\right)=\frac{(K+2)^{K+2}}{(K+1)^{K+1}},
$$

we conclude that

$$
\left\|\frac{\partial}{\partial z_{j}} f\right\|_{\delta} \leq \frac{(K+2)^{K+2}}{(K+1)^{K+1}} \frac{\delta}{\delta_{0}^{2}}\|f\|_{\delta_{0}}
$$

Finally, let $R: \mathbb{B}_{\delta_{0}} \subset \mathbb{C}^{K} \rightarrow \mathbb{C}^{K}$ be a bounded analytic 2-tail, denoted

$$
R\left(z_{1}, \ldots, z_{K}\right)=\left[\begin{array}{c}
R_{1}\left(z_{1}, \ldots, z_{K}\right) \\
\vdots \\
R_{K}\left(z_{1}, \ldots, z_{K}\right)
\end{array}\right] .
$$

Then

$$
\begin{aligned}
& \|D R(z)\|_{M, \delta}=\sup _{|z| \leq \delta} \sup _{|\eta|=1}|D R(z) \cdot \eta| \\
& =\sup _{|z| \leq \delta|\eta|=1} \sup _{\mid}\left|\left(\begin{array}{ccc}
\partial_{z_{1}} R_{1}(z) & \cdots & \partial_{z_{K}} R_{1}(z) \\
\vdots & \ddots & \vdots \\
\partial_{z_{1}} R_{K}(z) & \cdots & \partial_{z_{K}} R_{K}(z)
\end{array}\right)\left[\begin{array}{c}
\eta_{1} \\
\vdots \\
\eta_{K}
\end{array}\right]\right| \\
& \leq \sup _{|z| \leq \delta}\left(\begin{array}{c}
\left|\partial_{z_{1}} R_{1}(z)\right|+\ldots+\left|\partial_{z_{K}} R_{1}(z)\right| \\
\vdots \\
\left|\partial_{z_{1}} R_{K}(z)\right|+\ldots+\left|\partial_{z_{K}} R_{K}(z)\right|
\end{array}\right) \\
& \leq \max \left\{K \frac{(K+2)^{K+2}}{(K+1)^{K+1}} \frac{\delta}{\delta_{0}^{2}}\left\|R_{1}\right\|_{\delta_{0}}, \ldots, K \frac{(K+2)^{K+2}}{(K+1)^{K+1}} \frac{\delta}{\delta_{0}^{2}}\left\|R_{K}\right\|_{\delta_{0}}\right\} \\
& =\frac{K(K+2)^{K+2}}{(K+1)^{K+1}} \frac{\delta}{\delta_{0}^{2}}\|R\|_{\delta_{0}},
\end{aligned}
$$

where we have applied (55) to the components of $R$. 


\subsection{Parameterization method for stable and unstable man- ifolds of hyperbolic equilibria of vector fields}

Suppose that $g: \mathbb{R}^{K} \rightarrow \mathbb{R}^{K}$ is a real analytic vector field, that $g(\bar{y})=0$ and that $D g(\bar{y})$ has $K_{s}$ distinct eigenvalues with strictly negative real part. The case of $K_{s}<K$ is of greatest interest to us. Denote the eigenvalues by $\left\{\lambda_{i}^{s}\right\}_{i=1}^{K_{s}}$, so that $\operatorname{Re}\left(\lambda_{i}^{s}\right)<0$, and let $\left\{\xi_{i}^{s}\right\}_{i=1}^{K_{s}}$ be the associated eigenvectors. Let $\Lambda_{s}$ be the $K_{s} \times K_{s}$ matrix having $\left\{\lambda_{i}^{s}\right\}$ on the diagonal, and zeros elsewhere, and $A_{s}$ the $K \times K_{s}$ matrix whose columns are the stable eigenvectors $\left\{\xi_{i}^{s}\right\}$. Lastly, let $\phi: \mathbb{R}^{K} \times \mathbb{R} \rightarrow \mathbb{R}^{K}$ denote the flow generated by $g$, and $e^{\Lambda_{s} t} \theta$, with $\theta \in \mathbb{R}^{K_{s}}$, be the linear flow generated by the matrix $\Lambda_{s}$.

The parameterization method provides an analytic injection $P: \mathbb{B}_{\nu} \cap \mathbb{R}^{K_{s}} \rightarrow$ $\mathbb{R}^{K}, \nu>0$, such that

$$
P(0)=\bar{y}, \quad D P(0)=A_{s}
$$

and $P\left(\mathbb{B}_{\nu}\right) \subset W^{s}(\bar{y})$. The key observation is that if the parameterization $P$ satisfies

$$
\phi(P(\theta), t)=P\left(e^{\Lambda_{s} t} \theta\right) \quad \text { for all } \quad \theta \in \mathbb{B}_{\nu}
$$

then its image lies in the stable manifold. To see this let $\theta \in \mathbb{B}_{\nu}$, then

$$
\lim _{t \rightarrow \infty} \phi(P(\theta), t)=\lim _{t \rightarrow \infty} P\left(e^{\Lambda_{s} t} \theta\right)=P(0)=\bar{y} .
$$

We obtain a useful expression for $P$ by differentiating (57) with respect to time, and evaluating at zero, giving

$$
g[P(\theta)]=[D P(\theta)] \Lambda_{s} \theta \quad \text { for all } \theta \in \mathbb{B}_{\nu} .
$$

This is an equation involving only $P$, its derivative, the stable eigenvalues of $D g(\bar{y})$, and composition with the known vector field $g$. We refer to equation (58) as the invariance equation for the parameterization $P$.

In order to solve the invariance equation (58), constrained by the first order data $P(0)=\bar{y}$, and $D P(0)=A_{s}$, we assume that $P$ admits a power series representation

$$
P(\theta)=\sum_{|\alpha| \geq 0} a_{\alpha} \theta^{\alpha}
$$

Denote by $\left\{e_{i}\right\}_{i=1}^{K_{s}}$ the first order multi-indices

$$
e_{1}=(1,0, \ldots, 0) \quad \ldots \quad e_{K_{s}}=(0,0, \ldots, 1) .
$$

Using this notation we define the linear terms of $P$ to be $a_{(0, \ldots, 0)}=P(0)=\bar{y}$, and $a_{e_{i}}=\xi_{i}^{s}$. Recursion relations for the remaining coefficients can be obtained by substituting (59) into (58), expanding both sides as power series, and matching like powers of $\theta$. The resulting formal power series $P$ is referred to as the parameterization of $W^{s}(\bar{y})$ under $g$. We illustrate this computation explicitly in section 5 .

Remark 3.4. It is important to note that different scalings of the eigenvectors $\left\{\xi_{i}^{s}\right\}$ lead to different parameterizations of $W^{s}(\bar{y})$ under $g$. This non-uniqueness can be exploited in order to control the decay of the coefficients $a_{\alpha}$. 


\subsection{Numerical domain of the parameterization}

Let $P$ be a parameterization of $W^{s}(\bar{y})$ under $g$. As is made explicit in Section 2.2, we only ever compute and store a finite number of coefficients. Recursively solving (58) up to a fixed order $N$ results in a polynomial

$$
P_{N}(\theta)=\sum_{0 \leq|\alpha| \leq N} a_{\alpha} \theta^{\alpha}
$$

called the approximate parameterization. For fixed $N$ an essential step is to determine a domain $\mathbb{B}_{\nu} \cap \mathbb{R}^{K_{s}}$ on which $P_{N}$ is a sufficiently good approximation to $P$. The following definition makes this precise.

Definition 3.5. Let $\epsilon>0$ be a prescribed tolerance. The number $\nu>0$ is an $\epsilon$-numerical radius of validity for the approximate parameterization $P_{N}$ if

$$
\left\|g \circ P_{N}-\left[D P_{N}\right] \cdot \Lambda_{s}\right\|_{\nu} \leq \epsilon .
$$

Remark 3.6. In principle, numerical experimentation (made rigorous using interval arithmetic) can be used to select an appropriate value of $\nu$ directly from the definition. However, in practice once a reasonable guess for $\nu$ has been obtained by non-rigorous numerics, it can be more efficient to evaluate the $\nu$-weighted $\Sigma$-norm, providing a bound for the supremum norm. Specifically we expand $g\left(P_{N}(\theta)\right)$ and $\left[D P_{N}(\theta)\right] \Lambda_{s} \theta$ as power series $\sum_{|\alpha| \geq 0} A_{\alpha} \theta^{\alpha}$ and $\sum_{|\alpha| \geq 0} B_{\alpha} \theta^{\alpha}$, respectively. If $g$ is an $M$-th order polynomial vector field (as in the application in Section 5), then the composition $g \circ P_{N}$ is a polynomial, and the resulting sum has a finite number of terms. Define the error function

$$
\mathrm{E}(\theta)=g\left(P_{N}(\theta)\right)-\left[D P_{N}(\theta)\right] \Lambda_{s} \theta,
$$

and compute the $\nu$-weighted $\Sigma$-norm

$$
\|\mathrm{E}\|_{\Sigma, \nu}=\sum_{|\alpha| \geq 0}\left|A_{\alpha}-B_{\alpha}\right| \nu^{|\alpha|}
$$

If $\|\mathrm{E}\|_{\Sigma, \nu} \leq \epsilon$ then $\nu$ is an $\epsilon$-numerical radius of validity.

\section{A-Posteriori analysis of $\boldsymbol{P}_{N}$}

In this section we consider the convergence of the series expansion of $P$, derive bounds on the truncation error $\left\|P-P_{N}\right\|_{\nu}$ in terms of the numerically computed value of $\|\mathrm{E}\|_{\Sigma, \nu}$, and bound the decay rates of the parameterization coefficients $a_{\alpha}$. While the theorem presented in this section is stated for rigorous validation of the parameterization method for the stable manifold, the theorem can be used to validate unstable manifold computations by considering the flow in backward time. 


\subsection{Parameterized manifold validation theorem}

In order that the present section stand alone, we explicitly state all assumptions for the present validation theorem.

$\mathcal{P} .1$ Assume that $g=\left(g_{1}, \ldots, g_{K}\right): \mathbb{B}_{\rho} \subset \mathbb{C}^{K} \rightarrow \mathbb{C}^{K}$ is a bounded analytic vector field having $g(0)=0$ and $\operatorname{det}[D g(0)] \neq 0$.

$\mathcal{P} .2$ Assume that $D g(0)$ has $0<K_{s}<K$ distinct stable eigenvalues $\left\{\lambda_{i}^{s}\right\}_{i=1}^{K_{s}}$. Let $\left\{\xi_{i}^{s}\right\}_{i=1}^{K_{s}}$ denote the eigenvectors associated with the stable eigenvalues. We let $\Lambda_{s}$ denote the $K_{s} \times K_{s}$ diagonal matrix having the $\lambda_{i}^{s}$ on the diagonal, and $A$ be the matrix having the $\xi_{i}^{s}$ as columns.

$\mathcal{P} .3$ Assume that $P_{N}: \mathbb{B}_{\nu} \subset \mathbb{C}^{K_{s}} \rightarrow \mathbb{C}^{K}$ is a $N$-th order polynomial, with $N \geq 2$, having

$$
P_{N}(0)=0, \quad \text { and } \quad D P_{N}(0)=A,
$$

and which solves the equation

$$
g \circ P_{N}=D P_{N} \cdot \Lambda_{s}
$$

exactly to $N$-th order (in the sense that the power series coefficients of order $|\alpha| \leq N$ on the left are equal to those on the right).

Definition 4.1 (Validation values). The collection of positive constants $\nu, \epsilon_{\mathrm{tol}}$, $C_{1}, C_{2}, \rho^{\prime}, \rho$ and $\mu$ are validation values for $P_{N}$ if

1. $\left\|g \circ P_{N}-D P_{N} \cdot \Lambda_{s}\right\|_{\Sigma, \nu}<\epsilon_{\text {tol }}$;

2. $\left\|P_{N}\right\|_{\nu} \leq \rho^{\prime}<\rho$;

3. $\left\|D g\left[P_{N}\right]\right\|_{M, \nu} \leq C_{1}$;

4. $\max _{|\alpha|=2} \max _{1 \leq j \leq K}\left\|\partial^{\alpha} g_{j}\right\|_{\rho} \leq C_{2}$;

5. $\max _{1 \leq k \leq K_{s}} \operatorname{Re}\left(\lambda_{k}^{s}\right)<-\mu$.

To simplify the statement of the result we introduce the following notation. Let

$$
N_{g}=\max _{1 \leq j \leq K} \#\left\{(k, l) \mid 1 \leq k, l \leq K \text { such that } \partial^{k} \partial^{l} g_{j} \not \equiv 0\right\}
$$

be the maximum number of nontrivial elements in the Hessian of $g_{j}$. Clearly

$N_{g} \leq 2^{K}$, but for given $g$ a better bound may be found. Let $M_{1}$ and $M_{2}$ be positive numbers such that

$$
\begin{aligned}
& M_{1} \geq N_{g}, \\
& M_{2} \geq \frac{K(K+2)^{K+2}}{(K+1)^{K+1}} .
\end{aligned}
$$

Note that $M_{2} \geq 27 / 4$. 
Theorem 4.2. Given validation values $\nu, \epsilon_{\mathrm{tol}}, C_{1}, C_{2}, \rho, \rho^{\prime}$ and $\mu$, assume that $N$ and $\delta$ satisfy the three inequalities

$$
\begin{aligned}
N+1 & >\frac{C_{1}}{\mu}, \\
\delta & >\frac{2 \epsilon_{\text {tol }}}{(N+1) \mu-C_{1}}, \\
\delta & <\min \left\{\frac{(N+1) \mu-C_{1}}{C_{2} M_{1} M_{2}}, \frac{\rho-\rho^{\prime}}{K+2}\right\} .
\end{aligned}
$$

Then there is a unique parameterization function $P: \mathbb{B}_{\nu} \subset \mathbb{C}^{K_{s}} \rightarrow \mathbb{C}^{K}$ solving (57). In addition, the truncation error is bounded by

$$
\left\|P-P_{N}\right\|_{\nu} \leq \delta
$$

and the parameterization coefficients $a_{\alpha} \in \mathbb{C}^{K}$ decay as

$$
\left|a_{\alpha}\right| \leq \frac{\delta}{\nu^{|\alpha|}} \quad \text { for }|\alpha|>N
$$

The following three lemmata, whose proofs are presented in the next subsection, provide the core arguments for the proof of Theorem 4.2. Recall that $\mathcal{X}_{0}$ is the space of bounded analytic $N$-tails on $\mathbb{B}_{\nu}$.

Lemma 4.3. If $N+1>C_{1} / \mu$, then the linear operator $\mathfrak{L}: \mathcal{X}_{0} \rightarrow \mathcal{X}_{0}$ given by

$$
\mathfrak{L}(h)=D h \cdot \Lambda_{s}-D g\left[P_{N}\right] \cdot h
$$

is well-defined and invertible. Furthermore,

$$
\left\|\mathfrak{L}^{-1}\right\|_{\mathcal{X}_{0}} \leq \frac{1}{(N+1) \mu-C_{1}}
$$

Since $g$ is analytic and bounded on $\mathbb{B}_{\rho} \subset \mathbb{C}^{K}$, for each $|z| \leq \rho^{\prime}$ the function $g$ has a Taylor expansion at $z$ defined on a ball of radius at least $\delta_{0}=\rho-\rho^{\prime}$. Let $R_{z}: \mathbb{B}_{\delta_{0}} \subset \mathbb{C}^{K} \rightarrow \mathbb{C}^{K}$ denote the family of functions given by the second order Taylor remainders,

$$
g(z+\eta)=g(z)+D g(z) \cdot \eta+R_{z}(\eta) \quad \text { for }|\eta| \leq \delta_{0},
$$

hence $R_{z}=O\left(|\eta|^{2}\right)$ as $\eta \rightarrow 0$. For each $h \in \mathcal{X}_{0}$ define

$$
\begin{aligned}
R_{P_{N}}(h): \mathbb{B}_{\nu} & \rightarrow \mathbb{C}^{K} \\
\theta & \mapsto R_{P_{N}(\theta)}[h(\theta)] .
\end{aligned}
$$

Define the function $E: \mathbb{B}_{\nu} \subset \mathbb{C}^{K_{s}} \rightarrow \mathbb{C}^{K}$ by

$$
E(\theta)=g\left(P_{N}(\theta)\right)-D P_{N}(\theta) \cdot \Lambda_{s} \theta .
$$


Lemma 4.4. If $N+1>C_{1} / \mu$, then the operator $\Phi: \mathcal{X}_{0} \rightarrow \mathcal{X}_{0}$ given by

$$
\Phi(h)=\mathfrak{L}^{-1}\left[E+R_{P_{N}}(h)\right]
$$

is well-defined. Furthermore, $h$ is a fixed point of $\Phi$ if and only if

$$
g\left[P_{N}(\theta)+h(\theta)\right]=D\left[P_{N}(\theta)+h(\theta)\right] \cdot \Lambda_{s} \theta \quad \text { for all } \theta \in \mathbb{B}_{\nu} .
$$

Lemma 4.5. Under the hypotheses of Theorem 4.2 the operator $\Phi$ defined in Lemma 4.4 is a contraction mapping of the ball $U_{\delta}=\left\{h \in \mathcal{X}_{0}:\|h\|_{\nu} \leq \delta\right\}$ into itself.

Proof of Theorem 4.2: By Lemma 4.5 and the contraction mapping theorem, $\Phi$ has a unique fixed point $h \in U_{\delta}$. By Lemma 4.4, the function $P: \mathbb{B}_{\nu} \subset$ $\mathbb{C}^{K_{s}} \rightarrow \mathbb{C}^{K}$ defined by $P=P_{N}+h$ solves (57). In particular, since $h$ is a bounded analytic $N$-tail, the series expansion for $P$ converges. Furthermore, since $h \in U_{\delta}$,

$$
\left\|P-P_{N}\right\|_{\nu}=\|h\|_{\nu} \leq \delta .
$$

The decay rates of $a_{\alpha}$ for $|\alpha|>N$ follow by applying the Cauchy estimates to the series expansion of $h$.

\subsection{Proofs of the Lemmas}

The proof of Lemma 4.3 requires the following estimate for the composition of an $N$-tail with an exponential.

Lemma 4.6. Let $q: \mathbb{B}_{\nu} \subset \mathbb{C}^{K_{s}} \rightarrow \mathbb{C}^{K}$ be an analytic $N$-tail, and let $\left\{\lambda_{i}^{s}\right\}_{i=1}^{K_{s}}$ and $\Lambda_{s}$ be as in P.2 in Section 4.1, and let $\mu$ satisfy assumption 5 in Definition 4.1. Then

$$
\left\|q \circ e^{\Lambda_{s} t} \theta\right\|_{\nu} \leq e^{-(N+1) \mu t}\|q\|_{\nu}
$$

Proof. We write

$$
q\left(e^{\Lambda_{s} t} \theta\right)=\left[\begin{array}{c}
q_{1}\left(e^{\Lambda_{s} t} \theta\right) \\
\vdots \\
q_{K}\left(e^{\Lambda_{s} t} \theta\right)
\end{array}\right],
$$

where $q_{i}: \mathbb{B}_{\nu} \subset \mathbb{C}^{K_{s}} \rightarrow \mathbb{C}, 1 \leq i \leq K$. Note that

$$
e^{\Lambda_{s} t} \theta=e^{-\mu t}\left(\begin{array}{c}
e^{\left(\lambda_{1}^{s}+\mu\right) t} \theta_{1} \\
\vdots \\
e^{\left(\lambda_{k}^{s}+\mu\right) t} \theta_{k}
\end{array}\right)=e^{-\mu t} e^{\tilde{\Lambda}_{s} t} \theta,
$$

where $e^{\tilde{\Lambda}_{s} t}$ is a (non-strict) component-wise contraction, as

$$
\left|\left(e^{\tilde{\Lambda}_{s} t} \theta\right)_{i}\right|=e^{\left(\lambda_{i}^{s}+\mu\right) t}\left|\theta_{i}\right| \leq\left|\theta_{i}\right| \quad \text { for all } t \geq 0 \text { and } 1 \leq i \leq K .
$$

Then for $\theta \in \mathbb{B}_{\nu}$

$$
\left|q_{i}\left(e^{\Lambda_{s} t} \theta\right)\right|=\left|q_{i}\left(e^{-\mu t} e^{\tilde{\Lambda}_{s} t} \theta\right)\right| \leq e^{-(N+1) \mu t}\left\|q_{i} \circ e^{\tilde{\Lambda} t}\right\|_{\nu} \leq e^{-(N+1) \mu t}\left\|q_{i}\right\|_{\nu},
$$


where the first inequality follows by applying the inequality from Lemma 3.2 to the $N$-tail $q_{i}$, with $e^{-\mu t}$ as the scalar. Applying the previous estimate to the $K$ components of $q$ gives $\left\|q \circ e^{\Lambda_{s} t}\right\|_{\nu} \leq e^{-(N+1) \mu t}\|q\|_{\nu}$, as desired.

\section{Proof of Lemma 4.3:}

Beginning with any analytic $N$-tail $p \in \mathcal{X}_{0}$, our task is to find an analytic $N$-tail $q \in \mathcal{X}_{0}$ satisfying

$$
D q\left[\Lambda_{s}(\theta)\right]-D g\left[P_{N}(\theta)\right] \cdot q(\theta)=p(\theta), \quad \text { for all } \theta \in \mathbb{B}_{\nu} .
$$

We proceed by making a time-varying linear change of variables in Equation (70). With $\theta \in \mathbb{B}_{\nu}$ define

$$
\begin{aligned}
A(\theta, t) & =A(t)=D g\left[P_{N}\left(e^{\Lambda_{s} t} \theta\right)\right], \\
\bar{p}(\theta, t) & =\bar{p}(t)=p\left(e^{\Lambda_{s} t} \theta\right), \\
x(t) & =q\left[e^{\Lambda_{s} t} \theta\right] .
\end{aligned}
$$

Note that

$$
\frac{d}{d t} x(t)=D q\left[e^{\Lambda_{s} t} \theta\right] \Lambda_{s} e^{\Lambda_{s} t} \theta,
$$

so that $q$ solves (70) if and only if $x$ solves

$$
\frac{d}{d t} x-A(t) x=\bar{p}, \quad \text { for } t \geq 0 .
$$

This is a system of linear differential equations with analytic, non-constant coefficients. At $t=0$ we recover Equation (70).

We want to solve the linear differential equation by exploiting an integrating factor

$$
C(\theta, t)=C(t)=e^{-\int_{0}^{t} A(\theta, \tau) d \tau} .
$$

Note that $C(0)=I_{K \times K}$. We obtain the estimate, uniform in $\theta \in \mathbb{B}_{\nu}$,

$$
\begin{aligned}
\|C(t)\|_{M, \nu} & =\left\|e^{-\int_{0}^{t} A(s) d s}\right\|_{M, \nu} \\
& \leq e^{-\int_{0}^{t} A(s) d s \|_{M, \nu}} \\
& \leq e^{\int_{0}^{t}\|A(s)\|_{M, \nu} d s} \\
& =e^{\int_{0}^{t}\left\|D g \circ P_{n} \circ e^{\Lambda_{s} s}\right\|_{M, \nu} d s} \\
& \leq e^{C_{1} t} .
\end{aligned}
$$

Hence, for all $\theta \in \mathbb{B}_{\nu}$, using Lemma 4.6,

$$
\begin{aligned}
|C(\theta, t) \cdot \bar{p}(\theta, t)| & =\left|\left(e^{-\int_{0}^{t} A(\theta, s) d s}\right) \cdot\left(p\left[e^{\Lambda_{s} t} \theta\right]\right)\right| \\
& \leq e^{C_{1} t} \cdot\left(e^{-(N+1) \mu t}\|p\|_{\nu}\right) \\
& =e^{\left[C_{1}-(N+1) \mu\right] t}\|p\|_{\nu},
\end{aligned}
$$


so that for $N+1>C_{1} / \mu$ the argument of the exponential is negative, and the limit as $t \rightarrow \infty$ is zero. We now find that a solution of (71) is given by

$$
x(t)=-C^{-1}(t) \int_{t}^{\infty} C(s) \cdot \bar{p}(s) d s,
$$

implying that

$$
q(\theta)=x(0)=-\int_{0}^{\infty} C(s) \cdot \bar{p}(s) d s
$$

solves $(70)$.

Next, we establish that the function $q$ given by (73) is an analytic $N$-tail. Namely, it follows from Morera's theorem, using absolute integrability of the integrands $C(s) \cdot \bar{p}(s)$ on $[0, \infty)$ and $A(s)$ on $[0, t]$ for any $t>0$, that

1. $\left.\int_{0}^{t} A(\theta, s)\right) d s$ with $t \in[0, \infty)$,

2. $C(\theta, t)=e^{-\int_{0}^{t} A(\theta, s) d s}$ with $t \in[0, \infty)$,

3. and $q(\theta)$,

are analytic for $\theta \in \mathbb{B}_{\nu}$. Furthermore, $q(\theta)$ is an $N$-tail, since by computing its derivatives at $\theta=0$ up to $N$-th order, we find that they all vanish, as follows easily from the chain rule and the fact that $p$ is an $N$-tail.

Finally, we estimate, for $\theta \in \mathbb{B}_{\nu}$, using (72),

$$
\begin{aligned}
\left|\mathfrak{L}^{-1}[p](\theta)\right| & =|q(\theta)| \\
& =\left|-\int_{0}^{\infty} C(\theta, t) \cdot \bar{p}(\theta, t) d t\right| \\
& \leq \int_{0}^{\infty} e^{-\left[(N+1) \mu-C_{1}\right] t}\|p\|_{\nu} d t \\
& =\frac{1}{(N+1) \mu-C_{1}}\|p\|_{\nu},
\end{aligned}
$$

since $N+1>C_{1} / \mu$. The estimate (74) is uniform in $\theta \in \mathbb{B}_{\nu}$, hence this establishes

$$
\left\|\mathfrak{L}^{-1}\right\|_{\mathcal{X}_{0}} \leq \frac{1}{(N+1) \mu-C_{1}} .
$$

\section{Proof of Lemma 4.4:}

Note that $E$ is an analytic $N$-tail since the composition, difference, and differential of an analytic function is again analytic, and $E(0)=D E(0)=\ldots=$ $D^{N} E(0)=0$, as $P_{N}$ solves the invariance equation exactly to $N$-th order. Similarly, $R_{P_{N}} \circ h$ is the composition of analytic functions; and one can easily check that $R_{P_{N}}[h(0)]=\ldots=D^{N} R_{P_{N}}[h(0)]=0$ by the chain rule, as $h$ is an $N$-tail and $R_{P_{N}}$ is the second order Taylor remainder. Finally, recall that $\mathfrak{L}^{-1}$ is well defined and maps $N$-tails to $N$-tails whenever $N+1>C_{1} / \mu$. Hence $\Phi$ is an operator on $\mathcal{X}_{0}$. 
Now suppose that $h \in \mathcal{X}_{0}$ is a fixed point of $\Phi$, so that

$$
h(\theta)=\mathfrak{L}^{-1}\left[E(\theta)+R_{P_{N}} \circ h(\theta)\right] .
$$

This is equivalent to

$$
\begin{aligned}
D h(\theta) \cdot \Lambda_{s} \theta-D g\left[P_{N}(\theta)\right] \cdot h(\theta) & =E(\theta)+R_{P_{N}} \circ h(\theta) \\
& =g\left[P_{N}(\theta)\right]-D P_{N}(\theta) \cdot \Lambda_{s} \theta+R_{P_{N}} \circ h(\theta),
\end{aligned}
$$

so that

$$
D\left[P_{N}(\theta)+h(\theta)\right] \cdot \Lambda_{s} \theta=g\left[P_{N}(\theta)+h(\theta)\right]
$$

as desired.

\section{Proof of Lemma 4.5:}

We must establish that

(i) if $\delta$ is as in the hypotheses of Theorem 4.2, then $\Phi$ maps a $\delta$-neighborhood $U_{\delta}$ of the origin into itself;

(ii) there is a $\kappa<1$ so that for any $h_{1}, h_{2} \in U_{\delta}$ one has that $\left\|\Phi\left(h_{1}\right)-\Phi\left(h_{2}\right)\right\|_{\nu} \leq$ $\kappa\left\|h_{1}-h_{2}\right\|_{\nu}$.

We begin by explicitly and uniformly bounding $R_{z}(\eta)$, defined in (68), for $|z| \leq \rho^{\prime}$ and $|\eta|<\delta_{0}=\rho-\rho^{\prime}$. Let

$$
\mathcal{A}_{j}=\left\{\alpha:|\alpha|=2 \text { and } \partial^{\alpha} g_{j} \not \equiv 0\right\}, \quad j=1, \ldots, K,
$$

be the sets of nontrivial second derivatives of $g$. Then, by a straightforward counting argument, see (62),

$$
\sum_{\alpha \in \mathcal{A}_{j}} \frac{2}{\alpha !}=N_{g} \leq M_{1}
$$

where $\alpha !=\alpha_{1} ! \cdots \alpha_{K}$ !. Let $R_{z}=\left(R_{z}^{1}, \ldots, R_{z}^{K}\right)$, then Taylor's remainder theorem implies, for $1 \leq j \leq K$,

$$
\begin{aligned}
\left|R_{z}^{j}(\eta)\right| & =\left|\sum_{|\alpha|=2} \frac{2}{\alpha !} \eta^{\alpha} \int_{0}^{1}(1-t) \partial^{\alpha} g_{j}(z+t \eta) d t\right| \\
& \leq \sum_{|\alpha|=2} \frac{2}{\alpha !}|\eta|^{\alpha} \int_{0}^{1}(1-t)\left|\partial^{\alpha} g_{j}(z+t \eta)\right| d t \\
& \leq \sum_{|\alpha|=2, \alpha \in \mathcal{A}_{j}} \frac{2}{\alpha !}|\eta|^{2}\left\|\partial^{\alpha} g_{j}\right\|_{\rho} \\
& \leq M_{1} C_{2} \delta_{0}^{2} .
\end{aligned}
$$

Since this is uniform in $j$ we obtain

$$
\left\|R_{z}\right\|_{\delta_{0}} \leq M_{1} C_{2} \delta_{0}^{2}
$$


Proof of $(i)$ : Let $U_{\delta}=\left\{h \in \mathcal{X}_{0}:\|h\|_{\nu} \leq \delta\right\}$, and recall that $\delta_{0}=\rho-\rho^{\prime}$. For arbitrary, fixed $\theta \in \mathbb{B}_{\nu}$, let $z=P_{N}(\theta)$. Then $|z| \leq \rho^{\prime}$ by property 2 in Definition 4.1. For any $h \in U_{\delta}$ one has, using the bound from Lemma 3.2 as well as estimate (75),

$$
\left|R_{P_{N}(\theta)}^{j} h(\theta)\right|=\left|R_{z}^{j}(h(\theta))\right| \leq\left\|R_{z}\right\|_{\delta} \leq \frac{\delta^{2}}{\delta_{0}^{2}}\left\|R_{z}\right\|_{\delta_{0}} \leq C_{2} M_{1} \delta^{2},
$$

for all $j=1, \ldots, K$ and uniformly in $\theta \in \mathbb{B}_{\nu}$. Hence

$$
\begin{aligned}
\|\Phi(h)\|_{\nu} & =\left\|\mathfrak{L}^{-1}\left[E+R_{P_{N}}(h)\right]\right\|_{\nu} \\
& \leq\left\|\mathfrak{L}^{-1}\right\|_{\mathcal{X}_{0}}\left[\|E\|_{\nu}+\left\|R_{P_{N}}(h)\right\|_{\nu}\right] \\
& \leq \frac{1}{(N+1) \mu-C_{1}}\left[\epsilon_{\text {tol }}+C_{2} M_{1} \delta^{2}\right] \\
& =\frac{\epsilon_{\text {tol }}}{(N+1) \mu-C_{1}}+\frac{C_{2} M_{1}}{(N+1) \mu-C_{1}} \delta^{2} .
\end{aligned}
$$

Then in order that $\|\Phi(h)\|_{\nu}<\delta$, it is sufficient that

$$
\frac{\epsilon_{\text {tol }}}{(N+1) \mu-C_{1}}<\frac{\delta}{2}, \quad \text { and } \quad \frac{C_{2} M_{1}}{(N+1) \mu-C_{1}} \delta^{2}<\frac{\delta}{2} .
$$

These conditions are met, as we have hypothesized that inequalities (65) and (66) hold, and $M_{2} \geq 2$.

Proof of (ii): As in part $(i)$, for arbitrary, fixed $\theta \in \mathbb{B}_{\nu}$, let $z=P_{N}(\theta)$. Then $|z| \leq \rho^{\prime}$. Let $h_{1}, h_{2} \in U_{\delta}$. Using the mean value theorem, the bound from Lemma 3.3, which is applicable since $\delta<\delta_{0} /(K+2)$ by (66), as well as (63) and (75), we obtain

$$
\begin{aligned}
\left|R_{P_{N}(\theta)}^{j} h_{1}(\theta)-R_{P_{N}(\theta)}^{j} h_{2}(\theta)\right| & \leq\left|R_{z}^{j}\left(h_{1}(\theta)\right)-R_{z}^{j}\left(h_{2}(\theta)\right)\right| \\
& \leq\left\|D R_{z}\right\|_{M, \delta}\left\|h_{1}-h_{2}\right\|_{\nu} \\
& \leq \frac{M_{2} \delta}{\delta_{0}^{2}}\left\|R_{z}\right\|_{\delta_{0}}\left\|h_{1}-h_{2}\right\|_{\nu} \\
& \leq M_{2} \delta C_{2} M_{1}\left\|h_{1}-h_{2}\right\|_{\nu}
\end{aligned}
$$

for all $j=1, \ldots, K$ and uniformly in $\theta \in \mathbb{B}_{\nu}$. Hence

$$
\begin{aligned}
\left\|\Phi\left(h_{1}\right)-\Phi\left(h_{2}\right)\right\|_{\nu} & =\left\|\mathfrak{L}^{-1}\left[E+R_{P_{N}}\left(h_{1}\right)\right]-\mathfrak{L}^{-1}\left[E+R_{P_{N}}\left(h_{2}\right)\right]\right\|_{\nu} \\
& =\left\|\mathfrak{L}^{-1}\left[R_{P_{N}}\left(h_{1}\right)-R_{P_{N}}\left(h_{2}\right)\right]\right\|_{\nu} \\
& \leq\left\|\mathfrak{L}^{-1}\right\| \mathcal{X}_{0}\left\|R_{P_{N}}\left(h_{1}\right)-R_{P_{N}}\left(h_{2}\right)\right\|_{\nu} \\
& \leq \frac{1}{(N+1) \mu-C_{1}} C_{2} M_{2} M_{1} \delta\left\|h_{1}-h_{2}\right\|_{\nu} .
\end{aligned}
$$

Finally, let

$$
\kappa=\frac{C_{2} M_{2} M_{1}}{(N+1) \mu-C_{1}} \delta
$$

and note that $\kappa<1$, again by inequality (66). 


\section{Application: even homoclinics for Gray-Scott}

The goal of this section is to demonstrate the usefulness and applicability of our method by proving computationally the existence of multiple even homoclinic solutions in the Gray-Scott model

$$
\left\{\begin{array}{l}
u_{1}^{\prime \prime}=u_{1} u_{2}^{2}-\lambda\left(1-u_{1}\right) \\
u_{2}^{\prime \prime}=\frac{1}{\gamma}\left(u_{2}-u_{1} u_{2}^{2}\right)
\end{array}\right.
$$

which, when written as a vector field, has the form

$$
\left(\begin{array}{c}
\dot{v}_{1} \\
\dot{v}_{2} \\
\dot{v}_{3} \\
\dot{v}_{4}
\end{array}\right)=\left(\begin{array}{c}
v_{2} \\
v_{1} v_{3}^{2}-\lambda\left(1-v_{1}\right) \\
v_{4} \\
\frac{1}{\gamma}\left[v_{3}-v_{1} v_{3}^{2}\right]
\end{array}\right) .
$$

More specifically, we use the theory of the radii polynomials of Section 2, the theory of parameterization of invariant manifolds of Section 3 and the aposteriori analysis of Section 4 to prove Theorem 1.1, which we restate here.

Theorem 1.1. Fix $\bar{\gamma}=0.15$, and define

$$
\sigma_{k} \stackrel{\text { def }}{=}\left\{\begin{array}{cl}
\frac{3 k}{200}, & k=-8, \ldots,-1 \\
0, & k=0 \\
\frac{3 k}{100}, & k=1, \ldots, 22
\end{array}\right.
$$

and $\Sigma_{\bar{\gamma}} \stackrel{\text { def }}{=}\left\{\left(\frac{1+\sigma_{k}}{\bar{\gamma}}, \bar{\gamma}\right): k=-8, \ldots, 22\right\}$. Let $L=1.2$ for $k>0$ and $L=1.7$ for $k<0$. Then, for every $(\lambda, \gamma) \in \Sigma_{\bar{\gamma}}$ there exists a small set $B=B_{k} \subset$ $\mathbb{R}^{2} \times(C[0,1])^{2}$ containing a unique solution $\left(\tilde{\theta}, \tilde{u}_{1}, \tilde{u}_{2}\right)$ of problem $(10)$.

Note that each solution of Theorem 1.1 corresponds to an even homoclinic solution at the steady state $\bar{y}=(1,0,0,0)$ of the Gray-Scott vector field (76).

The proof of Theorem 1.1 is computer-assisted. The computational part of the proof requires success in the run of the Matlab program proof_Gray_Scott.m, which uses the interval arithmetic package Intlab developed in [31]. The code has three main parts. The first part concerns the verification of the hypotheses of Theorem 4.2, which provides us with rigorous bounds on the parameterization of the stable manifold of $\bar{y}=(1,0,0,0)$. In order to verify Theorem 4.2 , one needs to compute the validation values in Definition 4.1. This first requires computing explicitly the approximate parameterization $P_{N}(\theta)$. An explicit procedure for computing this approximation is presented in Section 5.1. Once the computation of $P_{N}(\theta)$ is completed, the second part of the program proof_Gray_Scott.m computes an approximate solution $\left(\hat{\theta}, \hat{u}_{h}\right)$ of $F^{(m, N)}=0$ given by (2.3), the Frechet derivative $D \Pi_{m} F\left(\hat{\theta}, \hat{u}_{h}\right)$, a numerical inverse $A_{m}^{\dagger}$ of $D \Pi_{m} F\left(\hat{\theta}, \hat{u}_{h}\right)$ and verifies that $A_{m}^{\dagger}$ is injective. The third part of the code concerns the construction of the radii polynomials and the verification of the hypotheses of Theorem 2.6. In 
Section 5.2, we present the final estimates specific for the construction of the radii polynomials of the Gray-Scott equation.

Once all the ingredients of the proof are in place, we present in Section 5.3 the proof of Theorem 1.1.

\subsection{Computation of the approximate parameterization $\boldsymbol{P}_{N}$ of the local stable manifold}

Let us first denote by $g: \mathbb{C}^{4} \rightarrow \mathbb{C}^{4}$ the function given by the right-hand side of (76). One has that at $\bar{y}=(1,0,0,0), D g(\bar{y})$ has two stable and two unstable eigenvalues. Denote the stable eigenvalues by $\lambda_{1}, \lambda_{2}$, and let $\xi_{1}, \xi_{2}$ be associated eigenvectors. As we will see, $\lambda_{1}$ and $\lambda_{2}$ are distinct, negative real numbers. Recall that the aim of the parameterization method is to find a ball $\mathbb{B}_{\nu}$ and smooth function $P: \mathbb{B}_{\nu} \subset \mathbb{R}^{2} \rightarrow \mathbb{R}^{4}$ which satisfies

$$
\begin{aligned}
P(0) & =\bar{y} \\
D P(0) & =\left[\xi_{1} \mid \xi_{2}\right], \\
g\left[P\left(\theta_{1}, \theta_{2}\right)\right] & =D P\left(\theta_{1}, \theta_{2}\right)\left(\begin{array}{cc}
\lambda_{1} & 0 \\
0 & \lambda_{2}
\end{array}\right)\left[\begin{array}{c}
\theta_{1} \\
\theta_{2}
\end{array}\right] .
\end{aligned}
$$

Assume that $P: \mathbb{B}_{\nu} \subset \mathbb{R}^{2} \rightarrow \mathbb{R}^{4}$ has the form

$$
P\left(\theta_{1}, \theta_{2}\right)=\left(\begin{array}{c}
\sum_{n=0}^{\infty} \sum_{m=0}^{\infty} a_{m n}^{1} \theta_{1}^{m} \theta_{2}^{n} \\
\sum_{n=0}^{\infty} \sum_{m=0}^{\infty} a_{m n}^{2} \theta_{1}^{m} \theta_{2}^{n} \\
\sum_{n=0}^{\infty} \sum_{m=0}^{\infty} a_{m n}^{3} \theta_{1}^{m} \theta_{2}^{n} \\
\sum_{n=0}^{\infty} \sum_{m=0}^{\infty} a_{m n}^{4} \theta_{1}^{m} \theta_{2}^{n}
\end{array}\right),
$$

where

$$
a_{00}^{i}=\bar{y}^{i} \quad a_{10}^{i}=\xi_{1}^{i} \quad a_{01}^{i}=\xi_{2}^{i} \quad \text { for } \quad i=1,2,3,4 .
$$

Note that in the set-up of the Introduction and Section 2, one has that

$$
P^{(0)}(\theta)=\left(\begin{array}{c}
\sum_{n=0}^{\infty} \sum_{m=0}^{\infty} a_{m n}^{1} \theta_{1}^{m} \theta_{2}^{n} \\
\sum_{n=0}^{\infty} \sum_{m=0}^{\infty} a_{m n}^{3} \theta_{1}^{m} \theta_{2}^{n}
\end{array}\right)
$$

and

$$
P^{(1)}(\theta)=\left(\begin{array}{c}
\sum_{n=0}^{\infty} \sum_{m=0}^{\infty} a_{m n}^{2} \theta_{1}^{m} \theta_{2}^{n} \\
\sum_{n=0}^{\infty} \sum_{m=0}^{\infty} a_{m n}^{4} \theta_{1}^{m} \theta_{2}^{n}
\end{array}\right) .
$$

We derive recursion relations by plugging the expansion given in (78) into the invariance equation (77), expanding, and matching like powers. To this end, 
note that the left-hand side of (77) becomes

$$
\begin{aligned}
& g\left[P\left(\theta_{1}, \theta_{2}\right)\right]=\left(\begin{array}{c}
P_{2}\left(\theta_{1}, \theta_{2}\right) \\
\left(P_{1} \cdot P_{3}^{2}\right)\left(\theta_{1}, \theta_{2}\right)+\lambda P_{1}\left(\theta_{1}, \theta_{2}\right)-\lambda \\
P_{4}\left(\theta_{1}, \theta_{2}\right) \\
\frac{1}{\gamma} P_{3}\left(\theta_{1}, \theta_{2}\right)-\frac{1}{\gamma}\left(P_{1} \cdot P_{3}^{2}\right)\left(\theta_{1}, \theta_{2}\right)
\end{array}\right), \\
& \sum_{n=0}^{\infty} \sum_{m=0}^{\infty} a_{m n}^{2} \theta_{1}^{m} \theta_{2}^{n} \\
&=\left(\begin{array}{c}
-\lambda+\sum_{n=0}^{\infty} \sum_{m=0}^{\infty} r_{m n} \theta_{1}^{m} \theta_{2}^{n}+\sum_{n=0}^{\infty} \sum_{m=0}^{\infty} \lambda a_{m n}^{1} \theta_{1}^{m} \theta_{2}^{n} \\
\sum_{n=0}^{\infty} \sum_{m=0}^{\infty} a_{m n}^{4} \theta_{1}^{m} \theta_{2}^{n} \\
\sum_{n=0}^{\infty} \sum_{m=0}^{\infty} \frac{1}{\gamma} a_{m n}^{3} \theta_{1}^{m} \theta_{2}^{n}-\sum_{n=0}^{\infty} \sum_{m=0}^{\infty} \frac{1}{\gamma} r_{m n} \theta_{1}^{m} \theta_{2}^{n}
\end{array}\right)
\end{aligned}
$$

where $\left\{r_{m n}\right\}$ are the coefficients of the power series expansion of the nonlinearity

$$
R\left(\theta_{1}, \theta_{2}\right)=\left(P_{1} \cdot P_{3}^{2}\right)\left(\theta_{1}, \theta_{2}\right) .
$$

Then

$$
\begin{aligned}
R\left(\theta_{1}, \theta_{2}\right) & =\sum_{n=0}^{\infty} \sum_{m=0}^{\infty} r_{m n} \theta_{1}^{m} \theta_{2}^{n} \\
& =\left(\sum_{n=0}^{\infty} \sum_{m=0}^{\infty} a_{m n}^{1} \theta_{1}^{m} \theta_{2}^{n}\right)\left(\sum_{n=0}^{\infty} \sum_{m=0}^{\infty} a_{m n}^{3} \theta_{1}^{m} \theta_{2}^{n}\right)^{2} \\
& =\left(\sum_{n=0}^{\infty} \sum_{m=0}^{\infty} a_{m n}^{1} \theta_{1}^{m} \theta_{2}^{n}\right)\left(\sum_{n=0}^{\infty} \sum_{m=0}^{\infty}\left[\sum_{j=0}^{n} \sum_{i=0}^{m} a_{(m-i)(n-j)}^{3} a_{i j}^{3}\right] \theta_{1}^{m} \theta_{2}^{n}\right) \\
& =\sum_{n=0}^{\infty} \sum_{m=0}^{\infty}\left(\sum_{j=0}^{n} \sum_{i=0}^{m} \sum_{k=0}^{j} \sum_{\ell=0}^{i} a_{(m-i)(n-j)}^{1} a_{(i-\ell)(j-k)}^{3} a_{\ell k}^{3}\right) \theta_{1}^{m} \theta_{2}^{n},
\end{aligned}
$$

or

$$
r_{m n}=\sum_{j=0}^{n} \sum_{i=0}^{m} \sum_{k=0}^{j} \sum_{\ell=0}^{i} a_{(m-i)(n-j)}^{1} a_{(i-\ell)(j-k)}^{3} a_{\ell k}^{3} .
$$

Expanding the right-hand side of (77) gives

$$
D P\left(\theta_{1}, \theta_{2}\right)\left(\begin{array}{c}
\lambda_{1} \theta_{1} \\
\lambda_{2} \theta_{2}
\end{array}\right)=\left(\begin{array}{l}
\lambda_{1} \theta_{1} \frac{\partial}{\partial \theta_{1}} P_{1}\left(\theta_{1}, \theta_{2}\right)+\lambda_{2} \theta_{2} \frac{\partial}{\partial \theta_{2}} P_{1}\left(\theta_{1}, \theta_{2}\right) \\
\lambda_{1} \theta_{1} \frac{\partial}{\partial \theta_{1}} P_{2}\left(\theta_{1}, \theta_{2}\right)+\lambda_{2} \theta_{2} \frac{\partial}{\partial \theta_{2}} P_{2}\left(\theta_{1}, \theta_{2}\right) \\
\lambda_{1} \theta_{1} \frac{\partial}{\partial \theta_{1}} P_{3}\left(\theta_{1}, \theta_{2}\right)+\lambda_{2} \theta_{2} \frac{\partial}{\partial \theta_{2}} P_{3}\left(\theta_{1}, \theta_{2}\right) \\
\lambda_{1} \theta_{1} \frac{\partial}{\partial \theta_{1}} P_{4}\left(\theta_{1}, \theta_{2}\right)+\lambda_{2} \theta_{2} \frac{\partial}{\partial \theta_{2}} P_{4}\left(\theta_{1}, \theta_{2}\right)
\end{array}\right)
$$


Each component of this vector field has the form

$$
\begin{aligned}
& \lambda_{1} \theta_{1} \frac{\partial}{\partial \theta_{1}} P_{I}\left(\theta_{1}, \theta_{2}\right)+\lambda_{2} \theta_{2} \frac{\partial}{\partial \theta_{2}} P_{I}\left(\theta_{1}, \theta_{2}\right) \\
&=\lambda_{1} \theta_{1} \frac{\partial}{\partial \theta_{1}} \sum_{n=0}^{\infty} \sum_{m=0}^{\infty} a_{m n}^{I} \theta_{1}^{m} \theta_{2}^{n}+\lambda_{2} \theta_{2} \frac{\partial}{\partial \theta_{2}} \sum_{n=0}^{\infty} \sum_{m=0}^{\infty} a_{m n}^{I} \theta_{1}^{m} \theta_{2}^{n} \\
&=\sum_{n=0}^{\infty} \sum_{m=1}^{\infty} m \lambda_{1} a_{m n}^{I} \theta_{1}^{m} \theta_{2}^{n}+\sum_{n=1}^{\infty} \sum_{m=0}^{\infty} n \lambda_{2} a_{m n}^{I} \theta_{1}^{m} \theta_{2}^{n},
\end{aligned}
$$

where $I=1,2,3,4$.

We match like powers in (80) and (82), isolate the $m n$-terms on the left-hand side of the equation, and put the lower order terms on the right-hand side. This computation leads to the system of equations

$$
\left(\begin{array}{cccc}
-\left(m \lambda_{1}+n \lambda_{2}\right) & 1 & 0 & 0 \\
a_{00}^{3} a_{00}^{3}+\lambda & -\left(m \lambda_{1}+n \lambda_{2}\right) & 2 a_{00}^{0} a_{00}^{3} & 0 \\
0 & 0\left(m \lambda_{1}+n \lambda_{2}\right) & 1 \\
-a_{00}^{3} a_{00}^{3} / \gamma & 0 & 1 / \gamma-2 a_{00}^{1} a_{00}^{3} / \gamma & -\left(m \lambda_{1}+n \lambda_{2}\right)
\end{array}\right)\left[\begin{array}{c}
a_{m n}^{1} \\
a_{m n} \\
a_{m n}^{3} \\
a_{m n}^{3 n}
\end{array}\right]=\left(\begin{array}{c}
0 \\
-s_{m n} \\
0 \\
\frac{1}{\gamma} s_{m n}
\end{array}\right),
$$

where $a_{00}^{3}=0$ in our case since $\bar{y}=(1,0,0,0)$, see $(79)$, and

$$
s_{m n}=\sum_{j=0}^{n} \sum_{i=0}^{m} \sum_{k=0}^{j} \sum_{\ell=0}^{i} \bar{a}_{(m-i)(n-j)}^{1} \bar{a}_{(i-\ell)(j-k)}^{3} \bar{a}_{\ell k}^{3},
$$

with

$$
\bar{a}_{\ell k}^{I}= \begin{cases}0 & \text { if } \ell=m \text { and } k=n, \\ a_{\ell k}^{I} & \text { otherwise }\end{cases}
$$

for $I=1,3$. Then $s_{m n}$ depends only on known quantities (terms of order lower than $m n$ ). Since the coefficients of $P$ are given to first order by (79), equation (84) can be solved recursively to any desired finite order, as long as the matrix in (84) can be inverted. Using this, one can then exactly compute the approximate parameterization $P_{N}(\theta)$. Note that if we let $a_{m n}=\left(a_{m n}^{1}, a_{m n}^{2}, a_{m n}^{3}, a_{m n}^{4}\right)$ and $b_{m n}=\left(0,-s_{m n}, 0, s_{m n} / \gamma\right)$, the recursion equations have the matrix form

$$
\left[D g(p)-\left(\lambda_{1} m+\lambda_{2} n\right) I\right] a_{m n}=b_{m n},
$$

which is sometimes called the homological equation for $P$.

Remark 5.1 (Non-Resonance). We note that the matrix in (85) is the characteristic matrix for $D g(p)$. The equation for the coefficient $a_{m n}$ can be solved uniquely as long as

$$
\lambda_{1} m+\lambda_{2} n \neq \lambda_{i} \quad \text { for } i=1,2 .
$$

Hence, for a given set of Gray-Scott parameters, we need to check that $\lambda_{1} / \lambda_{2}$ nor $\lambda_{2} / \lambda_{1}$ is an integer. 
More generally, the estimates in Section 4 show we only need to exclude resonances

$$
\sum_{i=1, \ldots, K_{s}} k_{i} \lambda_{i}=\lambda_{j}, \quad j=1, \ldots, K_{s},
$$

for $\sum k_{i} \leq N$, so that we can find, by the above algorithm, a polynomial $P_{N}$ solving (61) exactly to $N$-th order. Indeed, we note that, since the eigenvalues of $D g(\bar{y})$ are bounded in absolute value by $C_{1}$, and $-\mu$ is an upper bound on the real part of stable eigenvalues, it is straightforward to conclude that there can be no resonances for $\sum k_{i} \geq N+1>C_{1} / \mu$, see (64).

Finally, we note that the theory of Section 4 goes through even in the presence of a resonance. However in that case the form of $\Lambda_{s}$ satisfying the invariance equation (58) cannot be taken to be linear, and the resulting homological equations are more complex than in the present case.

Remark 5.2 (Validation Values for $P_{N}$ ). We note that Theorem 4.2 is applicable to the Gray-Scott vector field by considering the function $\bar{g}(x)=g(\bar{y}+x)$, $\bar{P}_{N}=P_{N}-\bar{y}$ where $g$ is the Gray-Scott field, $P_{N}$ is the approximation whose coefficients are determined by (85), and $\bar{y}$ is an equilibrium of Gray-Scott. Using the explicit form of the Gray-Scott vector field it is possible to verify by hand that

$$
C_{2}=2 \rho \max \left(1, \frac{1}{\gamma}\right)
$$

satisfies condition (4) of Definition 4.1. The remaining validation value conditions are verified rigorously using interval arithmetic.

Remark 5.3 (Properties of $P_{N}$ ). Having a closed form for the coefficients, as given by (85), is essential for computations as it provides the method for computing the approximation $P_{N}$ to any desired order. In addition, the closed form allows us to establish additional useful properties of the parameterization function $P_{N}$. For example note that if $\lambda_{1}, \lambda_{2}$ are real (as is the case for GrayScott at the parameters we are interested in), then the coefficients $a_{\alpha}$ are always real. Then we can conclude that $P$ is real analytic, despite the fact that the theory of Section 4 is developed entirely in terms of functions of several complex variables.

Similarly, it is possible to show that for Gray-Scott $a_{m n}=0$ whenever $n+m \geq 2$ and $n \geq m$. We exploit this fact numerically, in order to speed up the computation of $P_{N}$ (only non-zero coefficients need be computed). The assertion can be checked directly for $n+m=2$, and can be proved inductively for $n+m>2$ by taking into account that $a_{00}^{3}=a_{01}^{3}=a_{10}^{1}=0$. Note that invertibility of the matrix implies that a coefficient is zero if and only if the right-hand side of the equation is zero, and this happens only when $s_{m n}=0$.

\subsection{Radii polynomials for Gray-Scott}

In this section we explicitly compute the radii polynomials for the Gray-Scott equation. Recall that in the context of (8), one has that the right-hand-side of 
the general equation (1) is given by

$$
\Psi(u)=\Psi\left(u_{1}, u_{2}\right)=\left(\begin{array}{c}
u_{1} u_{2}^{2}-\lambda+\lambda u_{1} \\
\frac{1}{\gamma}\left(u_{2}-u_{1} u_{2}^{2}\right)
\end{array}\right) .
$$

We assume that we have verified the existence of validation values $\nu, \epsilon_{\mathrm{tol}}, C_{1}$, $C_{2}, \rho, \rho^{\prime}$ and $\mu$, and that $N, \nu$ and $\delta$ satisfy the hypotheses of Theorem 4.2, so that

$$
\left|a_{\alpha}^{(j)}\right| \leq \frac{\delta}{\nu^{|\alpha|}}, \quad \text { for }|\alpha|>N \text { and } j=0,1,
$$

and

$$
\left\|P_{N}-P\right\|_{\mathbb{B}_{\nu}} \leq \delta
$$

Moreover, let

$$
0<r_{*}<\nu-\max _{1 \leq j \leq n}\left|\hat{\theta}_{j}\right|
$$

be some chosen a priori bound on the radius $r$.

The construction of the radii polynomials introduced in Definition 2.5 requires the computation of the bound $Y$ satisfying (22) and (23), and the bound $Z$ satisfying (25) and (26). Note that most of their construction was done in the general setting in Section 2.4. However, as mention in Section 2.4.2, some estimates required for the construction of $Z$ are rather technical and are only presented in the context of the Gray-Scott equation. More specifically, the computations of the bounds $\Lambda^{(0)}, \Lambda^{(1)} \in \mathbb{R}_{+}^{2}$ satisfying (41) and (37), respectively, were not completed, and the expansions (38) and (48) and the associated bounds $\Gamma^{(\ell)}, \Gamma_{j}^{(\ell)}, \widetilde{\Gamma}_{j}^{(\ell)}$ and $\bar{\Gamma}_{i, k}^{(\ell)}$ satisfying (39), (43), (44) and (49) were not carried through in the general case.

\subsubsection{The bounds $\Lambda$}

We need the bounds $\Lambda^{(0)}, \Lambda^{(1)} \in \mathbb{R}_{+}^{2}$ satisfying

$$
\sum_{i=1}^{n} \sum_{l=1}^{n}\left|\sum_{\alpha \in \mathcal{S}_{i, l}} a_{\alpha}^{(j)} \alpha_{i}\left(\alpha_{l}-\delta_{i, l}\right) \sigma^{\alpha-e_{i}-e_{l}}\right| \leq \Lambda^{(j)}
$$

Defining

$$
\begin{aligned}
& \mathcal{S}_{i, l}^{(N)} \stackrel{\text { def }}{=} \mathcal{S}_{i, l} \cap\left\{\alpha \in \mathbb{N}^{n}|| \alpha \mid \leq N\right\}, \\
& \mathcal{S}_{i, l}^{(\infty)} \stackrel{\text { def }}{=} \mathcal{S}_{i, l} \cap\left\{\alpha \in \mathbb{N}^{n}|| \alpha \mid>N\right\},
\end{aligned}
$$

we can use (87) to split the sum in (89) as follows:

$$
\begin{aligned}
\left|\sum_{\alpha \in \mathcal{S}_{i, l}} a_{\alpha}^{(j)} \alpha_{i}\left(\alpha_{l}-\delta_{i, l}\right) \sigma^{\alpha-e_{i}-e_{l}}\right| \leq \mid & \sum_{\alpha \in \mathcal{S}_{i, l}^{(N)}} a_{\alpha}^{(j)} \alpha_{i}\left(\alpha_{l}-\delta_{i, l}\right) \sigma^{\alpha-e_{i}-e_{l}} \mid \\
& +\left(\sum_{\alpha \in \mathcal{S}_{i, l}^{(\infty)}} \alpha_{i}\left(\alpha_{l}-\delta_{i, l}\right) \frac{\delta}{\nu^{|\alpha|}}|\sigma|^{\alpha-e_{i}-e_{l}}\right) \mathbf{1},
\end{aligned}
$$


where $\mathbf{1}=(1,1, \cdots, 1) \in \mathbb{R}^{n}$ and where the first (finite) sum can be rigorously bounded using interval arithmetic and the second (infinite) sum can be bounded using analytic estimates, see below.

For brevity, in the following we restrict our attention to the case $n=2$ for the Gray-Scott system. For $j \in\{0,1\}$, one has that

$$
\begin{aligned}
& \sum_{i=1}^{2} \sum_{l=1}^{2}\left|\sum_{\alpha \in \mathcal{S}_{i, l}} a_{\alpha}^{(j)} \alpha_{i}\left(\alpha_{l}-\delta_{i, l}\right) \sigma^{\alpha-e_{i}-e_{l}}\right| \leq\left|\sum_{\alpha \in \mathcal{S}_{1,1}^{(N)}} a_{\alpha_{1}, \alpha_{2}}^{(j)} \alpha_{1}\left(\alpha_{1}-1\right) \sigma_{1}^{\alpha_{1}-2} \sigma_{2}^{\alpha_{2}}\right| \\
& +2\left|\sum_{\alpha \in \mathcal{S}_{2,1}^{(N)}} a_{\alpha_{1}, \alpha_{2}}^{(j)} \alpha_{1} \alpha_{2} \sigma_{1}^{\alpha_{1}-1} \sigma_{2}^{\alpha_{2}-1}\right|+\left|\sum_{\alpha \in \mathcal{S}_{2,2}^{(N)}} a_{\alpha_{1}, \alpha_{2}}^{(j)} \alpha_{2}\left(\alpha_{2}-1\right) \sigma_{1}^{\alpha_{1}} \sigma_{2}^{\alpha_{2}-2}\right| \\
& \quad+\sum_{\alpha \in \mathcal{S}_{1,1}^{(\infty)}} \alpha_{1}\left(\alpha_{1}-1\right) \frac{\left|\sigma_{1}\right|^{\alpha_{1}-2}\left|\sigma_{2}\right|^{\alpha_{2}}}{\nu^{\alpha_{1}+\alpha_{2}}} \delta \mathbf{1}+2 \sum_{\alpha \in \mathcal{S}_{2,1}^{(\infty)}} \alpha_{1} \alpha_{2} \frac{\left|\sigma_{1}\right|^{\alpha_{1}-1}\left|\sigma_{2}\right|^{\alpha_{2}-1}}{\nu^{\alpha_{1}+\alpha_{2}}} \delta \mathbf{1} \\
& \quad+\sum_{\alpha \in \mathcal{S}_{2,2}^{(\infty)}} \alpha_{2}\left(\alpha_{2}-1\right) \frac{\left|\sigma_{1}\right|^{\alpha_{1}}\left|\sigma_{2}\right|^{\alpha_{2}-2}}{\nu^{\alpha_{1}+\alpha_{2}}} \delta \mathbf{1},
\end{aligned}
$$

where $\mathbf{1}=(1,1) \in \mathbb{R}^{2}$. The first three (finite) sums on the right-hand side of (90) can be bounded using interval arithmetic. The chosen a priori bound (88) on $r$ guarantees that the existence of $0<\hat{\sigma}_{k}<1$ for $k=1$, 2 such that

$$
\left|\frac{\sigma_{k}}{\nu}\right| \leq \hat{\sigma}_{k}, \quad \text { for all } \sigma_{k} \in\left[\hat{\theta}_{k}-r_{*}, \hat{\theta}_{k}+r_{*}\right] .
$$

Hence, the last three (infinite) sums of the right-hand side of (90) can be bounded using

$$
\begin{gathered}
\sum_{\alpha \in \mathcal{S}_{1,1}^{(\infty)}} \alpha_{1}\left(\alpha_{1}-1\right) \frac{\left|\sigma_{1}\right|^{\alpha_{1}-2}\left|\sigma_{2}\right|^{\alpha_{2}}}{\nu^{\alpha_{1}+\alpha_{2}}} \\
\leq \frac{1}{\nu^{2}} \sum_{\alpha_{1}=2}^{N} \alpha_{1}\left(\alpha_{1}-1\right) \hat{\sigma}_{1}^{\alpha_{1}-2} \sum_{\alpha_{2}=N+1-\alpha_{1}}^{\infty} \hat{\sigma}_{2}^{\alpha_{2}} \\
\quad+\frac{1}{\nu^{2}}\left(\sum_{\alpha_{1}=N+1}^{\infty} \alpha_{1}\left(\alpha_{1}-1\right) \hat{\sigma}_{1}^{\alpha_{1}-2}\right)\left(\sum_{\alpha_{2}=0}^{\infty} \hat{\sigma}_{2}^{\alpha_{2}}\right) \\
=\frac{\hat{\sigma}_{2}^{N+1}}{\nu^{2} \hat{\sigma}_{1}^{2}\left(1-\hat{\sigma}_{2}\right)} \sum_{\alpha_{1}=2}^{N} \alpha_{1}\left(\alpha_{1}-1\right)\left(\frac{\hat{\sigma}_{1}}{\hat{\sigma}_{2}}\right)^{\alpha_{1}} \\
\quad+\frac{\left(\left(N^{2}-N\right) \hat{\sigma}_{1}^{2}+\left(2-2 N^{2}\right) \hat{\sigma}_{1}+N^{2}+N\right) \hat{\sigma}_{1}^{N-1}}{\nu^{2}\left(1-\hat{\sigma}_{1}\right)^{3}\left(1-\hat{\sigma}_{2}\right)}
\end{gathered}
$$


and similarly for $\alpha \in \mathcal{S}_{2,2}^{(\infty)}$ by interchanging the subscripts 1 and 2 , as well as

$$
\begin{gathered}
\sum_{\alpha \in \mathcal{S}_{2,1}^{(\infty)}} \alpha_{1} \alpha_{2} \frac{\left|\sigma_{1}\right|^{\alpha_{1}-1}\left|\sigma_{2}\right|^{\alpha_{2}-1}}{\nu^{\alpha_{1}+\alpha_{2}}} \\
\leq \frac{1}{\nu^{2}} \sum_{\alpha_{1}=1}^{N-1} \alpha_{1} \hat{\sigma}_{1}^{\alpha_{1}-1} \sum_{\alpha_{2}=N+1-\alpha_{1}}^{\infty} \alpha_{2} \hat{\sigma}_{2}^{\alpha_{2}-1} \\
\quad+\frac{1}{\nu^{2}}\left(\sum_{\alpha_{1}=N}^{\infty} \alpha_{1} \hat{\sigma}_{1}^{\alpha_{1}-1}\right)\left(\sum_{\alpha_{2}=1}^{\infty} \alpha_{2} \hat{\sigma}_{2}^{\alpha_{2}-1}\right) \\
=\frac{\hat{\sigma}_{2}^{N} \quad \sum_{\nu^{2} \hat{\sigma}_{1}\left(1-\hat{\sigma}_{2}\right)^{2}} \sum_{\alpha_{1}=1}^{N-1} \alpha_{1}\left[\left(N-\alpha_{1}\right)\left(1-\hat{\sigma}_{2}\right)+1\right]\left(\frac{\hat{\sigma}_{1}}{\hat{\sigma}_{2}}\right)^{\alpha_{1}}}{\quad+\frac{\left(N+\hat{\sigma}_{1}-N \hat{\sigma}_{1}\right) \hat{\sigma}_{1}^{N-1}}{\nu^{2}\left(1-\hat{\sigma}_{1}\right)^{2}\left(1-\hat{\sigma}_{2}\right)^{2}} .}
\end{gathered}
$$

Here the finite remaining sums can be evaluated using interval arithmetic. Using the above inequalities, one can easily compute $\Lambda^{(0)}, \Lambda^{(1)} \in \mathbb{R}_{+}^{2}$.

\subsubsection{The bounds $\Gamma$}

In order to obtain the expansion (38), denote $\hat{u}_{h}=\left(\hat{u}_{1}, \hat{u}_{2}\right), \tilde{u}=\left(\tilde{u}_{1}, \tilde{u}_{2}\right)$ and $\tilde{v}=\left(\tilde{v}_{1}, \tilde{v}_{2}\right)$. For $\Psi$ given by (86), one gets that $d=3$ and that

$$
D \Psi\left(\hat{u}_{h}+\tilde{u} r\right) \tilde{v}-D \Psi\left(\hat{u}_{h}\right)\left(\Pi_{h}\right)^{n} \tilde{v}=\mathrm{v}^{(1)}+\mathrm{v}^{(2)} r+\mathrm{v}^{(3)} r^{2},
$$

where

$$
\begin{aligned}
\mathrm{v}^{(1)} & =\left(\begin{array}{c}
\left(\hat{u}_{2}^{2}+\lambda\right)\left(I-\Pi_{h}\right) \tilde{v}_{1}+2 \hat{u}_{1} \hat{u}_{2}\left(I-\Pi_{h}\right) \tilde{v}_{2} \\
-\frac{1}{\gamma} \hat{u}_{2}^{2}\left(I-\Pi_{h}\right) \tilde{v}_{1}+\frac{1}{\gamma}\left(1-2 \hat{u}_{1} \hat{u}_{2}\right)\left(I-\Pi_{h}\right) \tilde{v}_{2}
\end{array}\right) \\
\mathrm{v}^{(2)} & =\left(\begin{array}{c}
2\left(\hat{u}_{2} \tilde{u}_{2} \tilde{v}_{1}+\hat{u}_{1} \tilde{u}_{2} \tilde{v}_{2}+\hat{u}_{2} \tilde{u}_{1} \tilde{v}_{2}\right) \\
-\frac{2}{\gamma}\left(\hat{u}_{2} \tilde{u}_{2} \tilde{v}_{1}+\hat{u}_{1} \tilde{u}_{2} \tilde{v}_{2}+\hat{u}_{2} \tilde{u}_{1} \tilde{v}_{2}\right)
\end{array}\right) \\
\mathrm{v}^{(3)} & =\left(\begin{array}{c}
\tilde{u}_{2}^{2} \tilde{v}_{1}+2 \tilde{u}_{1} \tilde{u}_{2} \tilde{v}_{2} \\
-\frac{1}{\gamma}\left(\tilde{u}_{2}^{2} \tilde{v}_{1}+2 \tilde{u}_{1} \tilde{u}_{2} \tilde{v}_{2}\right)
\end{array}\right) .
\end{aligned}
$$

To find upper bounds for the terms given in (40) and (42), we use interval arithmetic to compute, for any $k \in\{0, \ldots, m-1\}$, the right-hand side of each 
of the following component-wise inequalities:

$$
\begin{aligned}
& \int_{t_{k}}^{t_{k+1}}\left|\mathrm{v}^{(1)}(s)\right| d s \leq \\
& \left(\begin{array}{c}
\max _{s \in\left[t_{k}, t_{k+1}\right]}\left\{\left|\hat{u}_{2}^{2}(s)+\lambda\right|+2\left|\hat{u}_{1}(s) \hat{u}_{2}(s)\right|\right\} \\
(1 / \gamma) \max _{s \in\left[t_{k}, t_{k+1}\right]}\left\{\hat{u}_{2}^{2}(s)+\left|1-2 \hat{u}_{1}(s) \hat{u}_{2}(s)\right|\right\}
\end{array}\right)\left(t_{k+1}-t_{k}\right) \omega \\
& \int_{t_{k}}^{t_{k+1}}(1-s)\left|\mathrm{v}^{(1)}(s)\right| d s \leq \\
& \left(\begin{array}{c}
\max _{s \in\left[t_{k}, t_{k+1}\right]}\left\{(1-s)\left|\left(\hat{u}_{2}^{2}(s)+\lambda\right)\right|+2(1-s)\left|\hat{u}_{1}(s) \hat{u}_{2}(s)\right|\right\} \\
(1 / \gamma) \max _{s \in\left[t_{k}, t_{k+1}\right]}\left\{(1-s)\left|\hat{u}_{2}^{2}(s)\right|+(1-s)\left|1-2 \hat{u}_{1}(s) \hat{u}_{2}(s)\right|\right\}
\end{array}\right)\left(t_{k+1}-t_{k}\right) \omega \\
& \int_{t_{k}}^{t_{k+1}}\left|\mathrm{v}^{(2)}(s)\right| d s \leq \\
& \left(\begin{array}{c}
2 \\
2 / \gamma
\end{array}\right)\left[\max _{s \in\left[t_{k}, t_{k+1}\right]}\left\{\left|\hat{u}_{1}(s)\right|+2\left|\hat{u}_{2}(s)\right|\right\}\right]\left(t_{k+1}-t_{k}\right)(\omega+1)^{2} \\
& \int_{t_{k}}^{t_{k+1}}(1-s)\left|\mathrm{v}^{(2)}(s)\right| d s \leq \\
& \left(\begin{array}{c}
2 \\
2 / \gamma
\end{array}\right)\left[\max _{s \in\left[t_{k}, t_{k+1}\right]}\left\{(1-s)\left|\hat{u}_{1}(s)\right|+2(1-s)\left|\hat{u}_{2}(s)\right|\right\}\right]\left(t_{k+1}-t_{k}\right)(\omega+1)^{2} \\
& \int_{t_{k}}^{t_{k+1}}\left|\mathrm{v}^{(3)}(s)\right| d s \leq\left(\begin{array}{c}
3 \\
3 / \gamma
\end{array}\right)\left(t_{k+1}-t_{k}\right)(\omega+1)^{3} \\
& \int_{t_{k}}^{t_{k+1}}(1-s)\left|\mathrm{v}^{(3)}(s)\right| d s \leq\left(\begin{array}{c}
3 \\
3 / \gamma
\end{array}\right)\left[\left(t_{k+1}-t_{k}\right)\left(1-\frac{t_{k}+t_{k+1}}{2}\right)\right](\omega+1)^{3},
\end{aligned}
$$

The bounds $\Gamma^{(\ell)}, \Gamma_{j}^{(\ell)}$ and $\widetilde{\Gamma}_{j}^{(\ell)}$ can be derived from this directly. We then have all the ingredients to build the $V^{(1)}, V^{(2)}, V^{(3)} \in \mathbb{R}^{2 m+4}$ from (45) and then the construction of $Z_{1}, \ldots, Z_{n(m+2)}$ follows directly from (46).

Concerning the estimate $Z_{\infty}$, for the expansion (48) we find $\overline{\mathrm{v}}^{(\ell)}=\mathrm{v}^{(\ell)}$ for $\ell=2,3$, while

$$
\overline{\mathrm{v}}^{(1)}=\left(\begin{array}{c}
\left(\hat{u}_{2}^{2}+\lambda\right) \tilde{v}_{1}+2 \hat{u}_{1} \hat{u}_{2} \tilde{v}_{2} \\
-\frac{1}{\gamma} \hat{u}_{2}^{2} \tilde{v}_{1}+\frac{1}{\gamma}\left(1-2 \hat{u}_{1} \hat{u}_{2}\right) \tilde{v}_{2}
\end{array}\right) .
$$

From this we estimate

$$
\begin{aligned}
& \sup _{s \in\left[t_{k}, t_{k+1}\right]}\left|\overline{\mathrm{v}}^{(1)}(s)\right| \leq\left(\begin{array}{c}
\max _{s \in\left[t_{k}, t_{k+1}\right]}\left\{\left|\hat{u}_{2}^{2}(s)+\lambda\right|+2\left|\hat{u}_{1}(s) \hat{u}_{2}(s)\right|\right\} \\
(1 / \gamma) \max _{s \in\left[t_{k}, t_{k+1}\right]}\left\{\hat{u}_{2}^{2}(s)+\left|1-2 \hat{u}_{1}(s) \hat{u}_{2}(s)\right|\right\}
\end{array}\right)(\omega+1) \\
& \sup _{s \in\left[t_{k}, t_{k+1}\right]}\left|\overline{\mathrm{v}}^{(2)}(s)\right| \leq\left(\begin{array}{c}
2 \\
2 / \gamma
\end{array}\right)\left[\max _{s \in\left[t_{k}, t_{k+1}\right]}\left\{\left|\hat{u}_{1}(s)\right|+2\left|\hat{u}_{2}(s)\right|\right\}\right](\omega+1)^{2} \\
& \sup _{s \in\left[t_{k}, t_{k+1}\right]}\left|\overline{\mathrm{v}}^{(3)}(s)\right| \leq\left(\begin{array}{c}
3 \\
3 / \gamma
\end{array}\right)(\omega+1)^{3} .
\end{aligned}
$$


The bounds $\bar{\Gamma}_{i, k}^{(\ell)}$ in (49) are then computed using interval arithmetic. Finally, using (51), one can compute the expansion (50) and finalize the computation of the coefficients of the radii polynomials in Definition 2.5.

\subsection{Proof of Theorem 1.1}

Proof. Consider $(\lambda, \bar{\gamma}) \in \Sigma_{\bar{\gamma}}$ and fix the following quantities, where subscripts + and - distinguish the cases $k>0$ and $k<0$, respectively:

$L_{+}=1.2, L_{-}=1.7 \quad-$ time rescaling factor;

$N_{+}=13, N_{-}=15-$ approximate parameterization order;

$\nu=1.5-$ domain radius of the parameterization;

$r_{*}=.003-$ a priori upper bound satisfying (88);

$\omega=.01-$ weight of the tail of $B(r, \omega)$ given by $(21)$;

$\xi_{1}=\left(\begin{array}{c}0 \\ \frac{0}{0.2} \\ \sqrt{1+L_{ \pm}^{2} / \bar{\gamma}} \\ -\frac{1.2 L_{ \pm}}{\sqrt{\bar{\gamma}+L_{ \pm}^{2}}}\end{array}\right) \quad-$ eigenvector of length 1.2 associated to $\lambda_{1}=-\frac{L_{ \pm}}{\sqrt{\bar{\gamma}}} ;$

$\xi_{2}=\left(\begin{array}{c}\frac{1.2}{\sqrt{1+\lambda L^{2}}} \\ -\frac{1.2 \sqrt{\lambda} L_{ \pm}}{\sqrt{1+\lambda L_{ \pm}^{2}}} \\ 0 \\ 0\end{array}\right)-$ eigenvector of length 1.2 associated to $\lambda_{2}=-\sqrt{\lambda} L_{ \pm} ;$

As mentioned earlier, the proof requires success in the run of the Matlab computer program proof_Gray_Scott.m. This computer program uses the package Intlab developed in [31]. The program has three parts:

Part I. Using the non-resonance condition mentioned in Remark 5.1, solve the homological equation (85) up to order $N_{ \pm}$and then compute explicitly the coefficients $a_{\alpha}$ of the approximate parameterization $P_{N_{ \pm}}$. Note that one can use the symmetry conditions from the second part of Remark 5.3 to speed up this computation. Next, we need to compute the validation values from Definition 4.1. Using interval arithmetic, we compute $\epsilon_{\mathrm{tol}}, \rho^{\prime}$ and $C_{1}$. We compute $C_{2}$ using Remark 5.2 and the computation of $\mu$ is trivial. We let $M_{1}=3, M_{2}=\frac{186624}{3125}$ and $\rho=\rho^{\prime}+0.0001$. Using all the above mentioned computed quantities, one can find $\delta=\delta(\lambda)>0$ such that the hypotheses of Theorem 4.2 are satisfied.

Part II. Verify the assumptions $\mathcal{A} .1, \mathcal{A} .2, \mathcal{A} .3$ and $\mathcal{A}$.4 from Section 2.3. More precisely, apply first Newton's method to the finite dimensional reduction (17) to get a numerical approximation $\left(\hat{\theta}, \hat{u}_{h}\right)$. Then compute the Frechet derivative $D \Pi_{m} F\left(\hat{\theta}, \hat{u}_{h}\right)$ and an approximate inverse $A_{m}^{\dagger}$ of $D \Pi_{m} F\left(\hat{\theta}, \hat{u}_{h}\right)$. Finally, we verify that the matrix $A_{m}^{\dagger}$ is injective by showing that $\left\|I_{m}-A_{m}^{\dagger} D \Pi_{m} F\left(\hat{\theta}, \hat{u}_{h}\right)\right\|_{\infty}$ is less than one.

Part III. Combine the ingredients from Part I and Part II to construct the radii polynomials of Section 5.2. In the process, one needs to determine $0<\hat{\sigma}_{k}<1$ 
for $k=1,2$, such that (91) holds. Using these polynomials, find $r>0$ satisfying the hypotheses of Theorem 4.2 and verify that $r \leq r_{*}$. The set $B=B(\lambda)=\left(\hat{\theta}, \hat{u}_{h}\right)+B(r, \omega)$ then contains a unique zero of $F$ given by (14). This unique zero correspond to an even homoclinic solution to the steady state $\bar{y}=(1,0,0,0)$ of the Gray-Scott vector field (76).

The program proof_Gray_Scott.m performed successfully all of the three steps of the above algorithm with $m=1200$ for the parameter values $(\lambda, \bar{\gamma}) \in$ $\left\{\left(\frac{1+\sigma_{k}}{\bar{\gamma}}, \bar{\gamma}\right): k=-8, \ldots,-1\right\}$, and with $m=350$ for the parameter values $(\lambda, \bar{\gamma}) \in\left\{\left(\frac{1+\sigma_{k}}{\bar{\gamma}}, \bar{\gamma}\right): k=1, \ldots, 22\right\}$. The source code is available at [4].

\section{References}

[1] L. Ahlfors. Complex Analysis. McGraw-Hill; 3rd Edition, 1979.

[2] J.B. van den Berg, and J.-P. Lessard. Chaotic braided solutions via rigorous numerics: chaos in the Swift-Hohenberg equation. SIAM J. Appl. Dyn. Syst., 7(3):988-1031, 2008.

[3] J.B. van den Berg, J.-P. Lessard, and K. Mischaikow. Global smooth solution curves using rigorous branch following. Math. Comput., 79 (271), 1565-1584, 2010.

[4] J.B. van den Berg, J.-P. Lessard, J.D. Mireles James, and K. Mischaikow. proof_Gray_Scott.m, Matlab code for rigorous computation of connecting orbits in Gray-Scott. www.math.rutgers.edu/ jmireles/grayScottPage.html

[5] W.-J. Beyn. The numerical computation of connecting orbits in dynamical systems. IMA J. Numer. Anal., 10(3):379-405, 1990.

[6] B. Breuer, J. Horák, P. J. McKenna, and M. Plum. A computer-assisted existence and multiplicity proof for travelling waves in a nonlinearly supported beam. J. Differential Equations, 224(1):60-97, 2006.

[7] X. Cabré, E. Fontich, and R. de la Llave. The parameterization method for invariant manifolds. I. Manifolds associated to non-resonant subspaces. Indiana Univ. Math. J., 52(2):283-328, 2003.

[8] X. Cabré, E. Fontich, and R. de la Llave. The parameterization method for invariant manifolds. II. Regularity with respect to parameters. Indiana Univ. Math. J., 52(2):329-360, 2003.

[9] X. Cabré, E. Fontich, and R. de la Llave. The parameterization method for invariant manifolds. III. Overview and applications. J. Differential Equations, 218(2):444-515, 2005. 
[10] A. R. Champneys, and Yu. A. Kuznetsov. Numerical detection and continuation of codimension-two homoclinic bifurcations. Internat. J. Bifur. Chaos Appl. Sci. Engrg., 4(4):785-822, 1994.

[11] S. Day, J.-P. Lessard, and K. Mischaikow. Validated continuation for equilibria of PDEs. SIAM J. Numer. Anal., 45(4):1398-1424 (electronic), 2007.

[12] E. J. Doedel, and Mark J. Friedman. Numerical computation of heteroclinic orbits. J. Comput. Appl. Math., 26(1-2):155-170, 1989. Continuation techniques and bifurcation problems.

[13] E. J. Doedel, M. J. Friedman, and B. I. Kunin. Successive continuation for locating connecting orbits. Numer. Algorithms, 14(1-3):103-124, 1997. Dynamical numerical analysis (Atlanta, GA, 1995).

[14] M. J. Friedman, and E. J. Doedel. Numerical computation and continuation of invariant manifolds connecting fixed points. SIAM J. Numer. Anal., 28(3):789-808, 1991.

[15] M. J. Friedman, and E. J. Doedel. Computational methods for global analysis of homoclinic and heteroclinic orbits: a case study. J. Dynam. Differential Equations, 5(1):37-57, 1993.

[16] M. Gameiro, J.-P. Lessard, and K. Mischaikow. Validated continuation over large parameter ranges for equilibria of PDEs. Math. Comput. Simulation, 79(4):1368-1382, 2008.

[17] M. Gameiro, and J.-P. Lessard. Analytic estimates and rigorous continuation for equilibria of higher-dimensional PDEs. J. Differential Equations, 249(9):2237 2268, 2010.

[18] M. Gameiro, and J.-P. Lessard. Rigorous computation of smooth branches of equilibria for the three dimensional Cahn-Hilliard equation. To appear in Numer. Math., 2010.

[19] P. Gray, and S.K. Scott, Autocatalytic reactions in the isothermal, continuous stirred tank reactor: Oscillations and instabilities in the system $A+2 B \rightarrow 3 B, B \rightarrow C$. Chem. Engrg. Sci., 39:1087-1097, 1984.

[20] A. Guillamon, and G. Huguet. A computational and geometric approach to phase resetting curves and surfaces. SIAM J. Appl. Dyn. Syst., 8(3):1005$1042,2009$.

[21] V. R. Korostyshevskiy, and T. Wanner. A Hermite spectral method for the computation of homoclinic orbits and associated functionals. J. Comput. Appl. Math., 206(2):986-1006, 2007.

[22] S. Krantz. Function Theory of Several Complex Variables. AMS Chelsea Publishing, 2nd Edition, 2001. 
[23] J. Hale, L.A. Peletier, and W.C. Troy. Exact homoclinic and heteroclinic solutions of the Gray-Scott model for autocatalysis. SIAM J. Appl. Math. 61 (2000), no. 1, 102-130 (electronic).

[24] Y. Hiraoka. Construction of approximate solutions for rigorous numerics of symmetric homoclinic orbits. In Workshops on "Pattern Formation Problems in Dissipative Systems" and "Mathematical Modeling and Analysis for Nonlinear Phenomena", RIMS Kôkyûroku Bessatsu, B3, pages 11-23. Res. Inst. Math. Sci. (RIMS), Kyoto, 2007.

[25] D.E. Knuth The Art of Computer Programming. Vol. 2, Seminumerical Algorithms, Addison-Wesley Publishing Co., Reading Mass. 1981.

[26] J.-P. Lessard. Recent advances about the uniqueness of the slowly oscillating periodic solutions of Wright's equation. J. Differential Equations, 248 (5): 992-1016, 2010.

[27] J.-D. Mireles James, and Hector Lomelí. Computation of heteroclinic arcs for the volume preserving Hénon map. SIAM J. Appl. Dyn. Syst., 9 (3): 919-953, 2010.

[28] M. T. Nakao. Numerical verification methods for solutions of ordinary and partial differential equations. Numer. Funct. Anal. Optim., 22(3-4):321356, 2001.

[29] S. Oishi. A numerical method of proving the existence and inclusion of connecting orbits for continuous dynamical systems. J. UCS, 4(2):193-201, 1998.

[30] C. Robinson. Dynamical Systems. Stability, symbolic dynamics, and chaos, CRC Press, 2nd Edition, 1999.

[31] S.M. Rump. INTLAB - INTerval LABoratory. In Tibor Csendes, editor, Developments in Reliable Computing, pages 77-104. Kluwer Academic Publishers, Dordrecht, 1999. http://www.ti3.tu-harburg.de/rump/.

[32] M. H. Schultz. Spline analysis. Prentice-Hall Inc., Englewood Cliffs, N.J., 1973. Prentice-Hall Series in Automatic Computation.

[33] D. Wilczak. Symmetric heteroclinic connections in the Michelson system: a computer assisted proof. SIAM J. Appl. Dyn. Syst., 4(3):489-514 (electronic), 2005.

[34] D. Wilczak, and P. Zgliczyński. Heteroclinic connections between periodic orbits in planar restricted circular three-body problem - a computer assisted proof. Comm. Math. Phys., 234(1): 37-75, 2003.

[35] N. Yamamoto. A numerical verification method for solutions of boundary value problems with local uniqueness by Banach's fixed-point theorem. SIAM J. Numer. Anal., 35(5):2004-2013 (electronic), 1998. 
[36] P. Zgliczyński. Covering relations, cone conditions and the stable manifold theorem. J. Differential Equations, 246(5):1774-1819, 2009. 